\title{
Exposed Pedagogy: Architecture as (a) Medium (of communication)
}

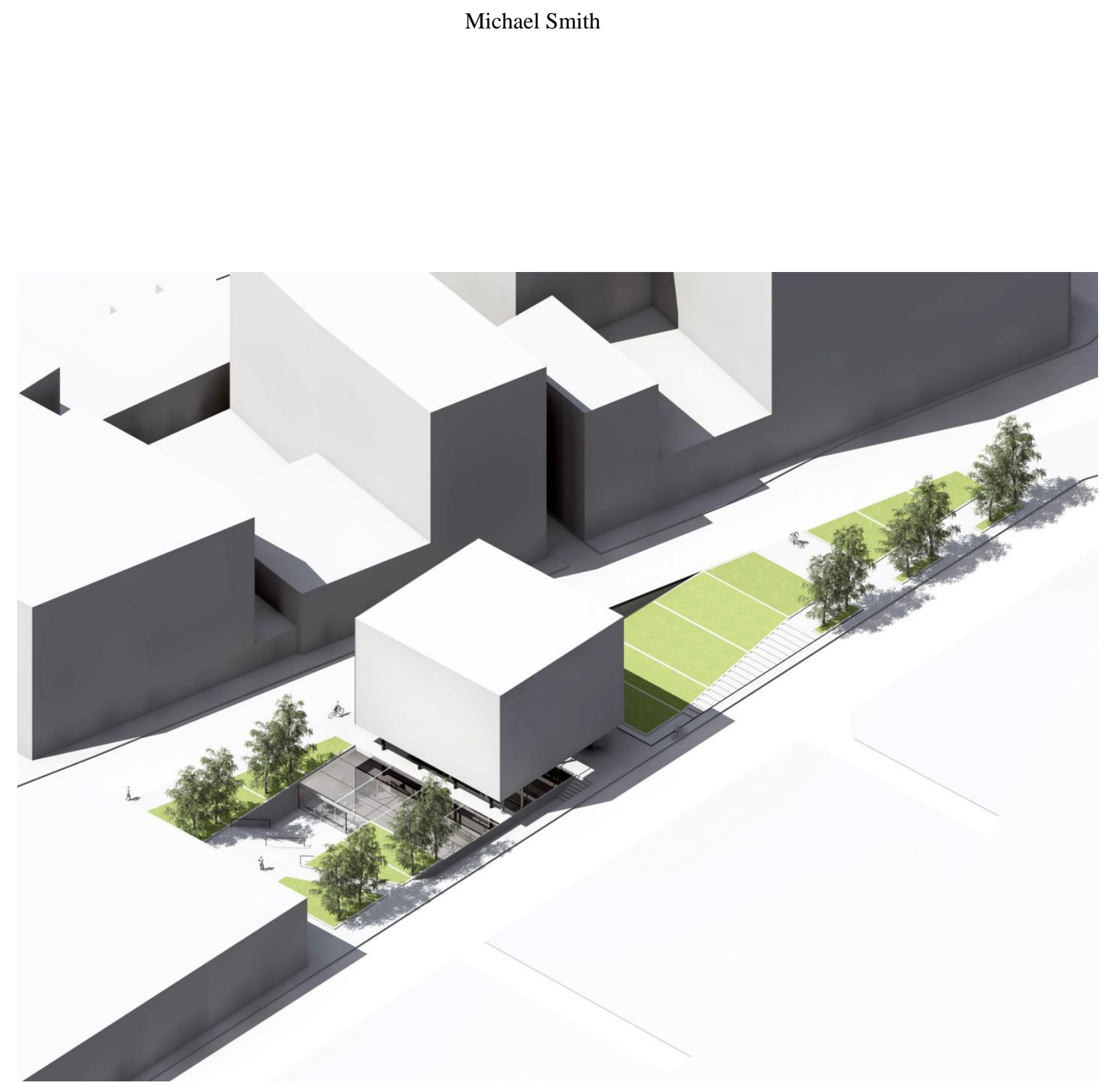

A 90 point thesis submitted to the Faculty of Architecture and Design, Victoria University of Wellington, in the fulfilment of the requirements for the degree of Masters of Architecture.

Victoria University of Wellington 


\begin{abstract}
Architecture schools are often isolated from the profession and the public they serve both pedagogically and physically, and often this is justified. Schools are not typically very public. However, schools could play a much greater role in the stimulation of a public discourse around architecture. The intent of this thesis is to examine how architecture can stimulate that discussion and ultimately how architecture could frame that discourse. The thesis proposes that an architecture school can and should be a vehicle for public discourse by way of framing that discourse on the one hand and overtly stimulating it on the other. Specifically, an architecture school has the capacity to contribute to public discourse through the visual affects of form making and the social implications of that same form. The research is divided into three components that chronicle an argument from contextualisation, through investigation to application.
\end{abstract}




\section{Acknowledgements}

To my parents Alistair and Karen

To my 2012 Class

To my supervisor Sam 


\section{Table of Contents}

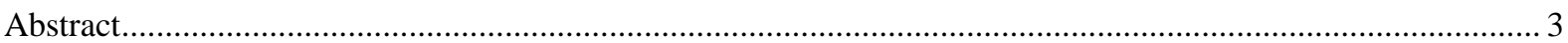

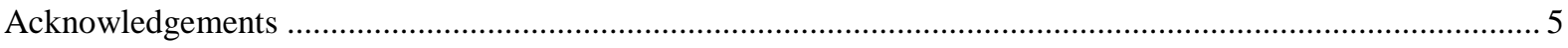

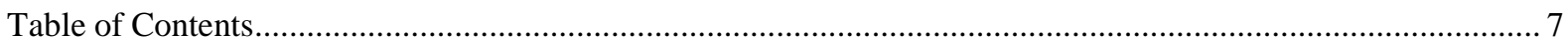

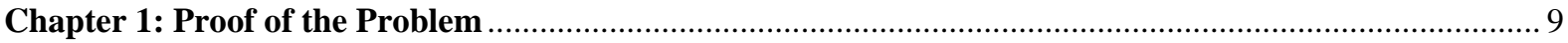

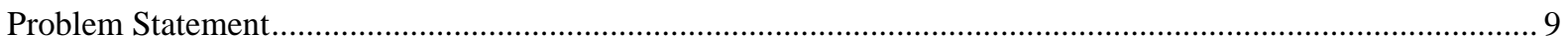

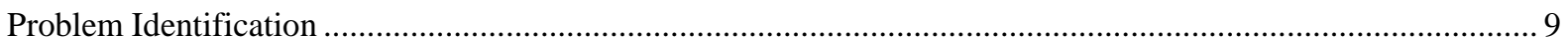

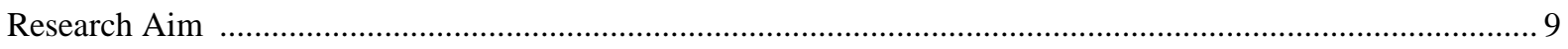

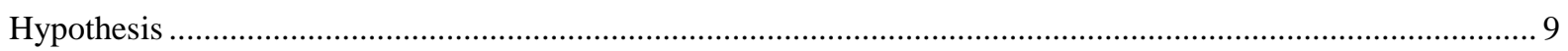

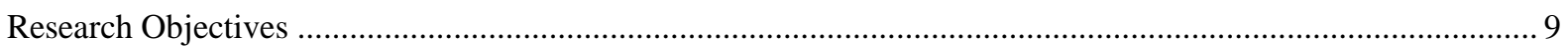

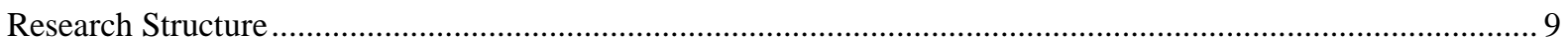

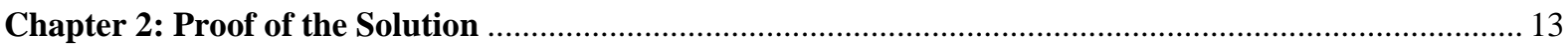

Solution Statement.

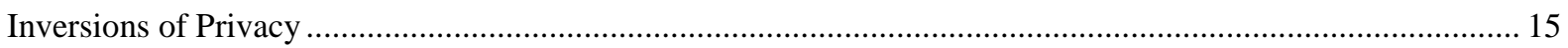

Architecture as Art.

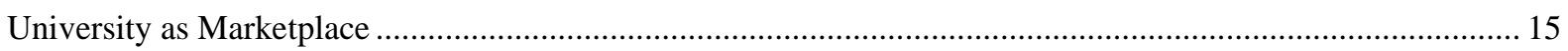

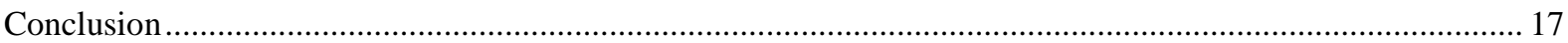

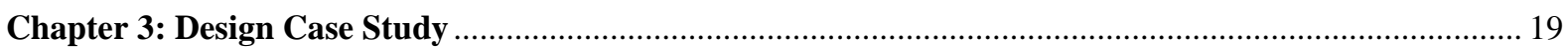

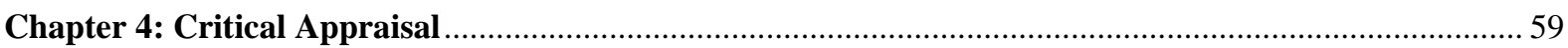

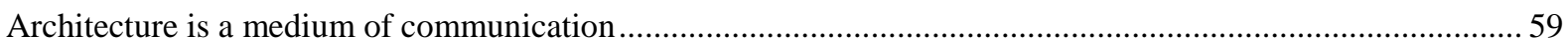

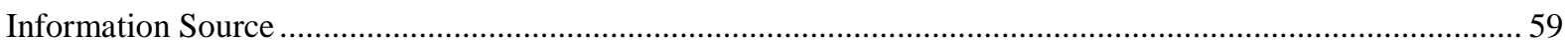

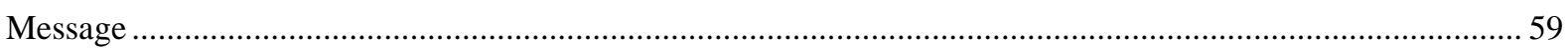

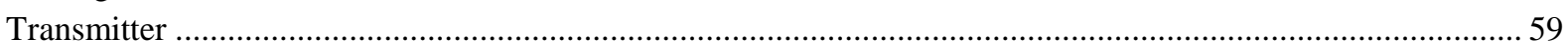

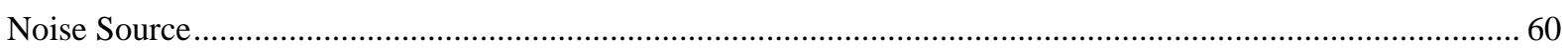

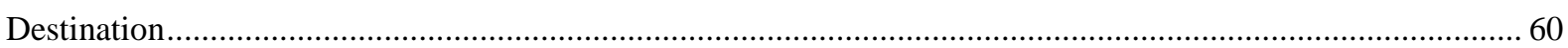

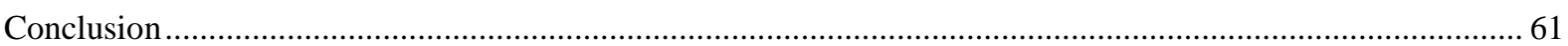

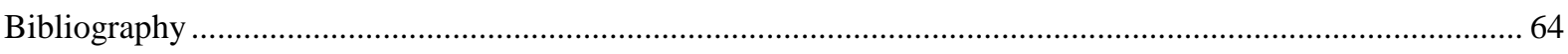

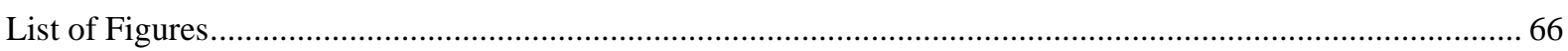


[1]

\section{Proof of the Problem}

\subsection{Problem Statement}

The facilities in an architecture school that are predominately designated as public are often not easily accessible, and consequently there is a sense from the public that they are trespassing. The problem is that the public facilities where discourse is at its most public within a school are not genuinely public. Citizens of the city should feel like those spaces are equally 'theirs' and not just 'belonging' to the students. The discourse is not framed for public engagement, and therefore, more often than not, it fails to stimulate any public discourse on architecture.

\subsection{Problem Identification}

The facilities in an architecture school that are designated as public do not accommodate the public because:

1. An architecture school establishes a distinct boundary between the territorial interior and the genuinely public exterior. The public facilities are internally located and have been physically and visually disconnected from the street. The problem is that there are no prominent connections between the interior and the exterior

2. An architecture school, more often than not, rejects loitering. Loitering is essential to ensure that the public inhabit a building for a prolonged period of time. Protracted inhabitation will enable an individual to become acquainted with the internal activities of a building and can encourage that inhabitant to interact with the building. Protracted interaction stimulates discourse.

\subsection{Research Aim}

The aim of this research is to establish design strategies that will enable an architecture school to stimulate a broader public discussion and frame that discourse.

\subsection{Hypothesis}

The publication of architectural output has been utilised extensively throughout the history of architecture in order to stimulate architectural discussion and frame that discourse. The discourse occurs primarily through published media in the form of journals and books (online and offline), stimulated by built work and unbuilt proposals. However, architecture is itself a medium of communication. The thesis proposes that the reconfiguration of architecture as a media output will provide the creative opportunity to propel the introverted spatial conditions of an architecture school into the public sphere.

\subsection{Research Objectives}

The thesis consists of four primary objectives:

1. Identify, describe and analyse the physical characterises that will enable an architecture school to operate as a medium of communication.

2. Identify how an architecture school can be physically and psychologically integrated into society.

3. Identify how can the sitting, mass, form and organisation of an architecture school can contribute toward a broader public discourse on architecture?

4. Formulate the results and conclusions into a framework.

\subsection{Research Structure}

The research is divided into three components that chronicle an argument from contextualisation, through investigation to application. In order to establish how an architecture school can be framed for public engagement and stimulate a broader public discourse the thesis critically explores three formal strategies. The strategies that are proposed identify how to produce a successful public space that will enable an architecture school to actively engage the public. In order to identify how the strategies can be implemented they are applied to a theoretical design. The theoretical design is an architecture school that has been amalgamated with a park. The design component of the thesis is utilised as a device to examine the applicability of the proposed solution. The results that are devised during the design component of the thesis are formulated into a framework. 


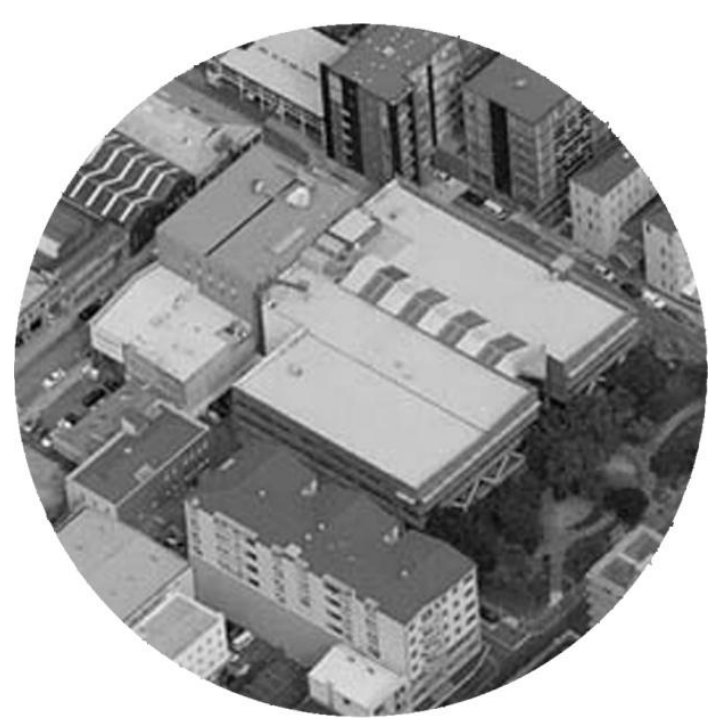

Wellington School of Architecture

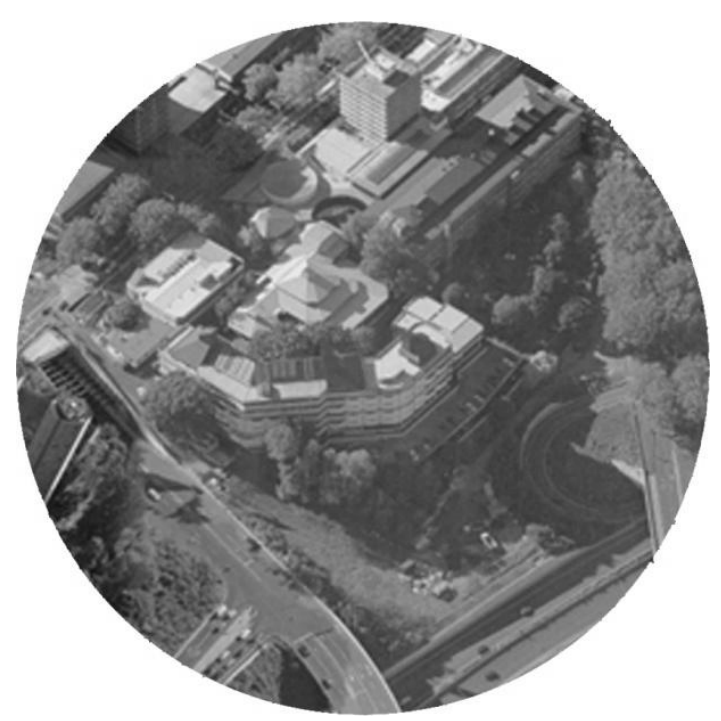

Auckland School of Architecture

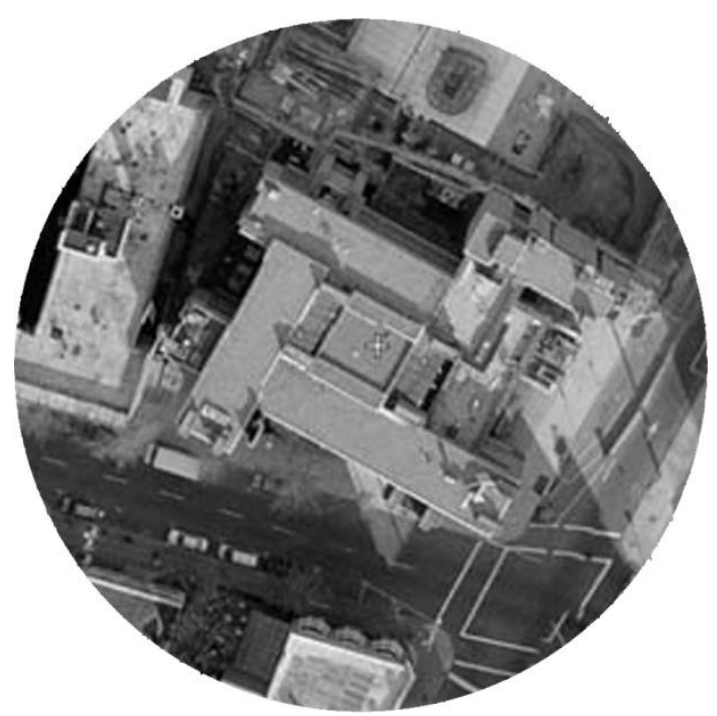

Fig 1.1] Building axonometric that depicts the segregated site conditions of an architecture school: Author adapted from, Bing Maps

Yale School of Architecture 


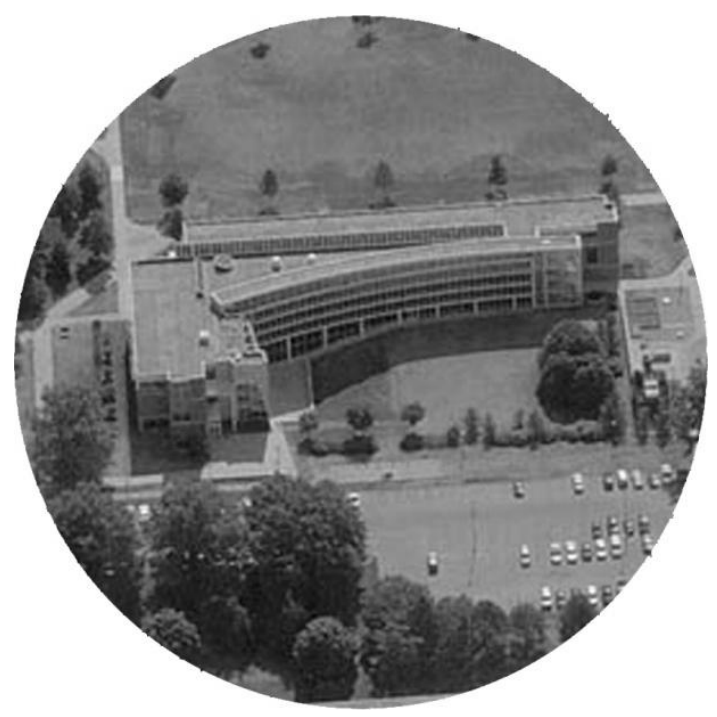

Minnesota School of Architecture

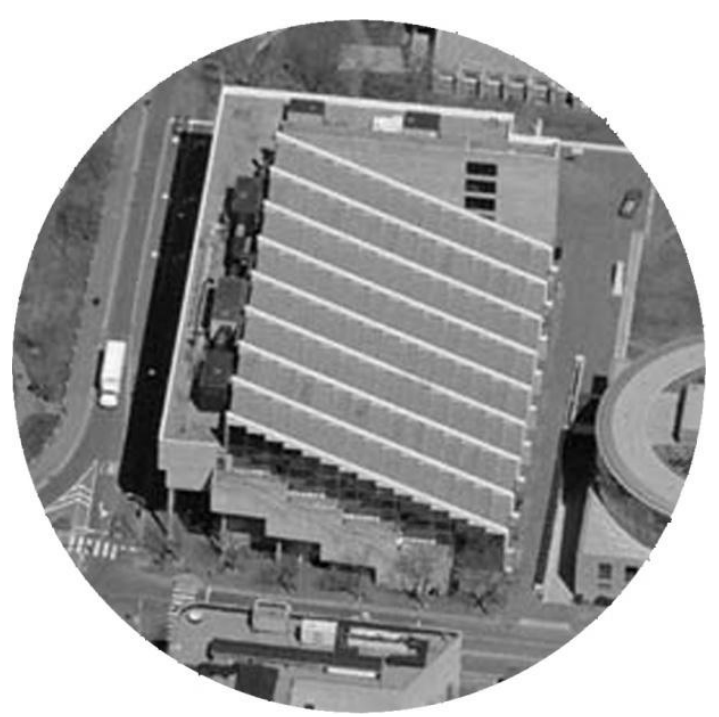

Harvard School of Architecture

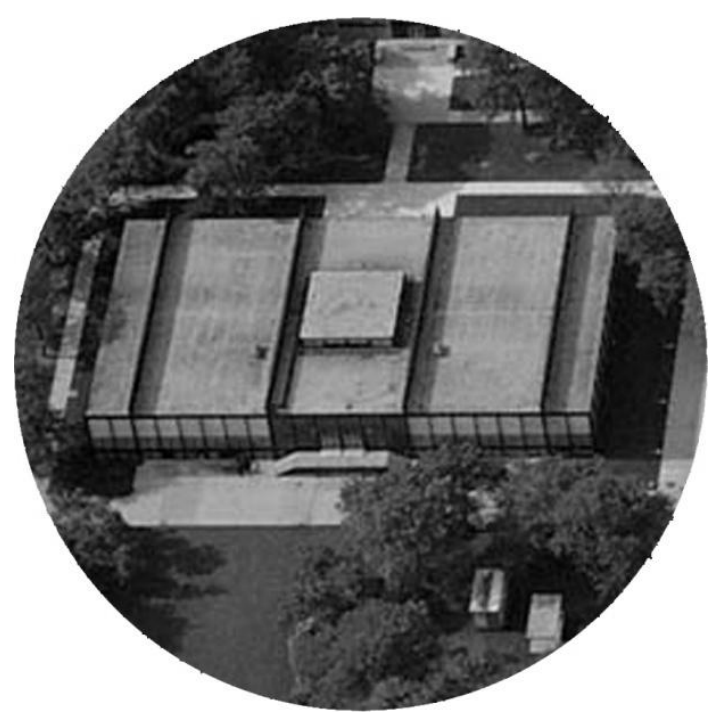

Fig 1.2] Building axonometric that depicts the segregated site conditions of an architecture school: Author adapted from, Bing Maps

Illinois Institute of Technology 


\section{$[2]$}

\section{Proof of the Solution}

\subsection{Proof of the Solution}

\section{Architecture as Medium - Space for Exchange}

The previous chapter stated that an architecture school is not framed for public engagement, and therefore, more often than not, it fails to stimulate any public discourse on architecture. In this chapter three strategies that will 'engage' public interaction are discussed. The intent of the strategies is to identify how architecture can stimulate a broader public discussion and frame that discourse.

\section{Inversions of Privacy} (Architecture must accommodate loitering).

In order for an architecture school to stimulate discourse it must facilitate loitering. Loitering is the act of remaining in a particular public place for a protracted time. Protracted inhabitation will enable an individual to become acquainted with the internal activities of a building and can encourage that inhabitant to interact with the building. ${ }^{1}$ Protracted interaction stimulates discourse.

The previous chapter identified that accessibility of an architecture school is, more often than not, isolated from the public. Protracted interaction does not occur because the architecture school establishes a physical distinction between 'inside' and 'outside'. The stimulation of discourse occurs 'inside' and is not accessible. Facilitation of free loitering does not occur.

In order to destabilise the distinction between 'inside' and 'outside' and facilitate loitering the building needs to operate as a continuation of the surrounding context. If the building is an extension of the surrounding context the citizens in the city will feel free to inhabit that space and through that inhabitation become acquainted with the internal activities. In the text 'Herman Hertzberger - Space and the Architect', it is identified that in order for a building to operate as an extension of the surrounding context the form should be perceived as an assemblage of components and be integrated into the city itself. ${ }^{2}$
A successful example in which this has been achieved is the Carpenter Centre designed by Le Corbusier in 1963. The Carpenter Centre for the Visual Arts is located on Harvard University's campus and is positioned between two parallel streets. In order to connect the two streets a single promenade penetrates the building. The promenade connects the interior with the campus and sidewalk. The centralized ramp permits a slow ascent through the interior in order to allow inhabitants visual and physical access. The centralized ramp injects the exterior into the interior. Inhabitation and loitering is encouraged because the promenade is an extension of the footpath and operates as a thoroughfare between two points. The footpath is a genuinely public space. The Carpenter Centre is an assemblage of components that has been integrated into the city itself.

The Kunsthal building designed by OMA in Rotterdam is another successful example in which architecture dissolves the distinction between 'outside' and 'inside'. The previous example identified how a thoroughfare can be used to construct a space that is both internal and external. The Kunsthal is an example that identifies how form can dissolve the perception of trespassing.

The interior of most buildings is predominately isolated from the exterior. Inhabitation results from a conscious decision to enter the interior. The Kunsthal identifies how architecture can subliminally stimulate engagement as opposed to requiring the public to initiate the engagement. Subliminal engagement dissolves the perception of trespassing and facilitates prolonged loitering. The Kunsthal is divided into three autonomous programs: an exhibition space, an auditorium and a restaurant. The autonomous programs that are located on different floors are connected by sloping floor planes and a series of tightly organized ramps that provide seamless connection between the interior and the exterior footpath. The slopping floors and ramps operate as a device that dissolves a definitive threshold. The definitive threshold is dissolved because the building does not contain an area that has been overtly expressed as an entrance.

The two buildings are successful examples in which the interior and exterior as well as public and private are intertwined. Inhabitation simultaneously occurs inside and outside. Architecture, more often than not, is unable to force interaction. The two 


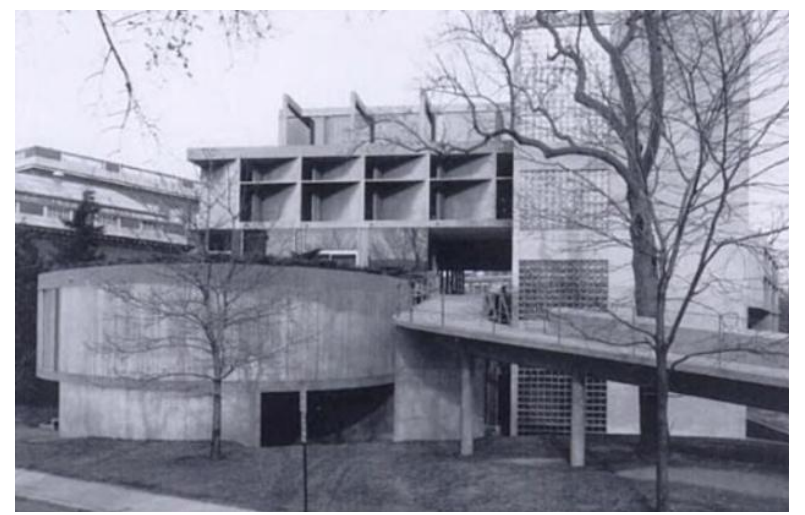

Fig2.1] Carpenter Centre

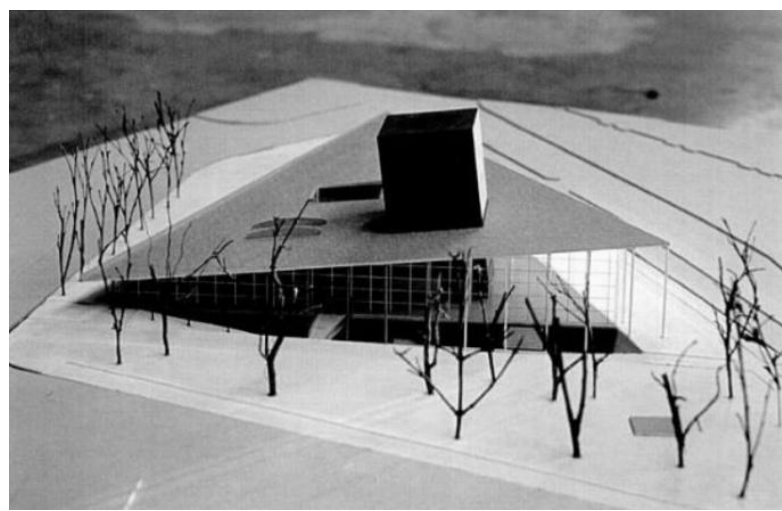

Fig2.2] Kunsthal Model

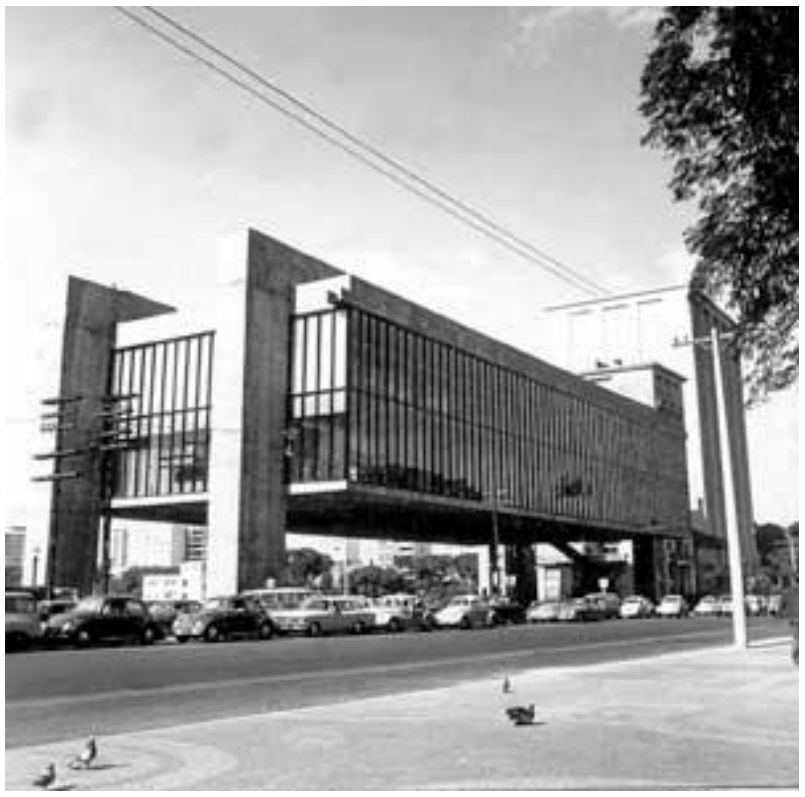

Fig2.3]The Museum de Arte de São Paulo 
buildings subliminally engage the public as opposed to requiring the public to initiate the engagement.

\section{Architecture as Art \\ (Architecture must reject autonomy).}

In the text ' Jane Rendell - Art and Architecture: A Place Between' Rendell proposes that art is a form of critical spatial practice that can transform architecture into a space of social critique. ${ }^{3}$ This transformation ensures that architecture is the subject of a highly concentrated gaze in which selfreflection, critical thinking and social change is stimulated. At present the discourse in an architecture school is not framed for public engagement, and therefore, more often than not, it fails to stimulate any public discourse on architecture. In order to frame architecture as art and stimulate a broader public discourse an architecture school should reject autonomy and operate as an interactive instillation.

A successful example in which this has been achieved is Brazilian Modernism. The Museum on the Seashore (1951), Museu de Arte de São Paulo (1960), and Cais das Artes (2008), are three examples of Brazilian Modernism in which architecture overtly operates as art.

The previous chapter established that an architecture school is ordinarily isolated from public consciousness. In order for architecture to stimulate discourse it must be experienced in a state of concentration. The Museu de Arte de São Paulo (1960) designed by Lina Bo Bardi and the Cais das Artes (2008) designed by Paulo Mendes da Rocha identify how architecture can frame the attention of an individual. In figure 2.3 and figure 2.5 the building mass is physically elevated above the ground. The area below the elevated mass is a genuinely public space and directs an observers' vision toward a specific point. Concentration is induced because there is a distinct visual disparity between the elevated mass and the surrounding context.

The Museum on the Seashore (1951) is another successful example in which architecture is art. The previous example identified how architecture can frame the attention of an individual. The Museum on the Seashore is an example that identifies how architecture can stimulate self-reflection, critical thinking and social change.
The Museum on the Seashore (1951) designed by Lina Bo Bardi is formally configured as a sculpture that is isolated in space. Jane Rendell alludes to the notion that art and architecture are typically differentiated in terms of their relationship to function. ${ }^{4}$ In figure 2.4 the building does not communicate a specific function. The building formally resembles a sculpture as opposed to the traditional conception of architecture. The sculpture stimulates a specific interaction that architecture is ordinarily not subject to. The sculpture is the subject of a highly concentrated gaze that is able to stimulate self-reflection and critical thinking.

The Museum on the Seashore (1951), Museu de Arte de São Paulo (1960), and Cais das Artes (2008), are three examples of Brazilian Modernism in which architecture overtly operates as art. Art shifts the experience of architecture that occurs in a state of distraction to a state that occurs through heightened concentration.

\section{University as Marketplace \\ (Architecture must operate as an accessible marketplace).}

At present, universities are segregated from society. The individual spaces are isolated from the public and the discourse that is stimulated in those spaces is restricted. In the text: 'Alexander, Christopher A Pattern Language' Alexander proposes that a university should operate as a market place in order to expose the stimulation of discourse. If an

architecture school is reconfigured as a marketplace the form will not operate as an isolated object; rather, the form and the surrounding context will be intertwined. Integration will expose the introverted spatial conditions and provide uninhibited access to the interior. The discourse that is internally stimulated will literally unfold in the city.

A successful example in which this has been achieved is the Architecture and Urbanism University at São Paulo designed by João Batista Vilanova Artigas. The building is physically elevated above the ground and is serviced by a series of interlinking ramps. The ramps enable the exterior to penetrate the interior an establish a sense of continuity. The public is freely permitted to engage the building. The internal discourse simultaneously occurs inside and outside. 


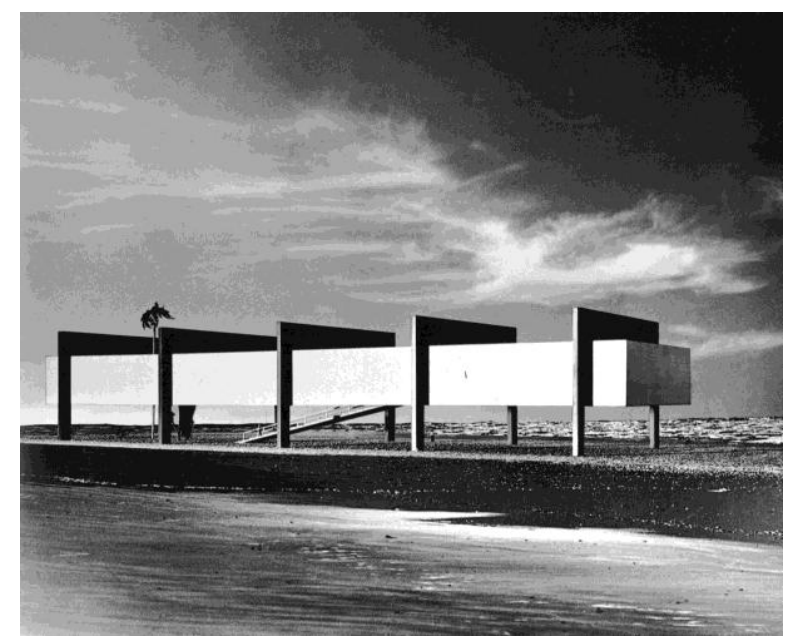

Fig2.4] Museum on the Seashore

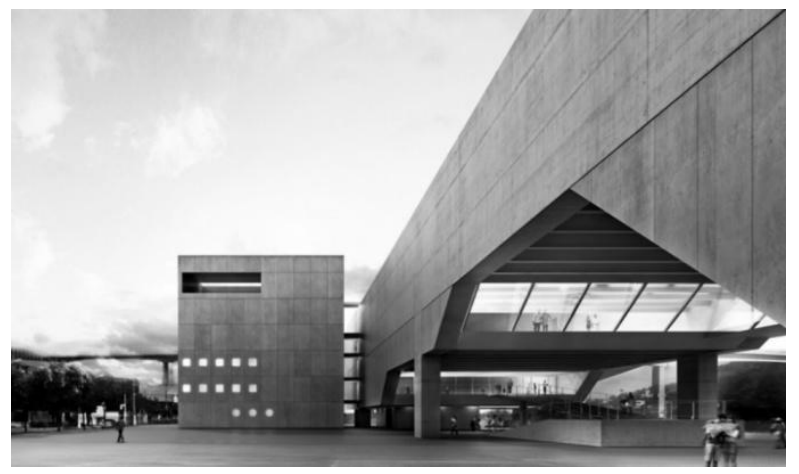

Fig2.5] Cais das Artes

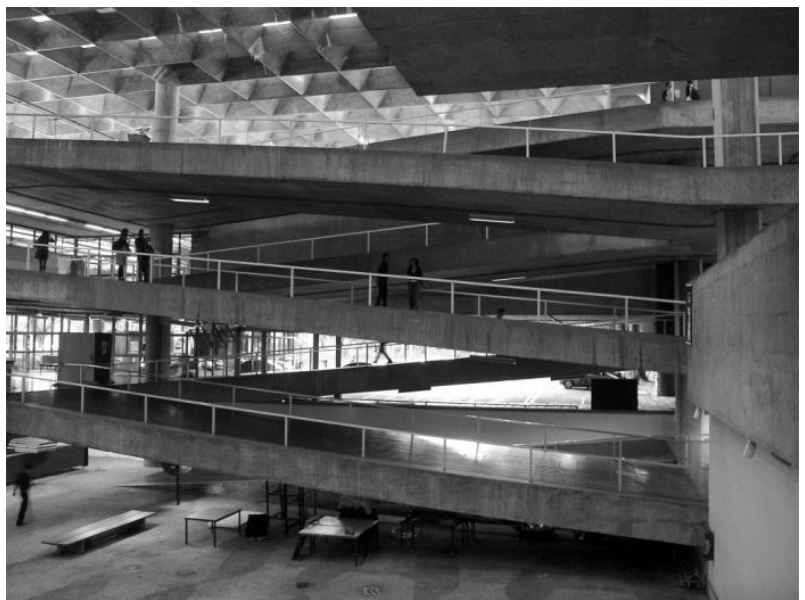

Fig2.6] College of Architecture and Urbanism of the University of São Paulo 


\section{Conclusion}

Each of the above strategies identify how the medium of architecture is able to be framed for public engagement and stimulate discourse. The three strategies have been summarised into the follow three descriptions:

1. Architecture must accommodate loitering.

2. Architecture must reject autonomy

3. Architecture must operate as an accessible marketplace.

1 Alexander, Christopher. A Pattern Language: Towns, Buildings, Construction. New York: Oxford Univ. Press, 1979. Print. P.495

2 Hertzberger, Herman, and Herman Hertzberger. Lessons for Students in Architecture: 2. Rotterdam: Press, 2000. Print. P.218

3 Rendell, Jane. Art and Architecture: A Place between. London [u.a.: I. B. Tauris, 2006. Print. P.4

Ibid P.4 


\section{Design}

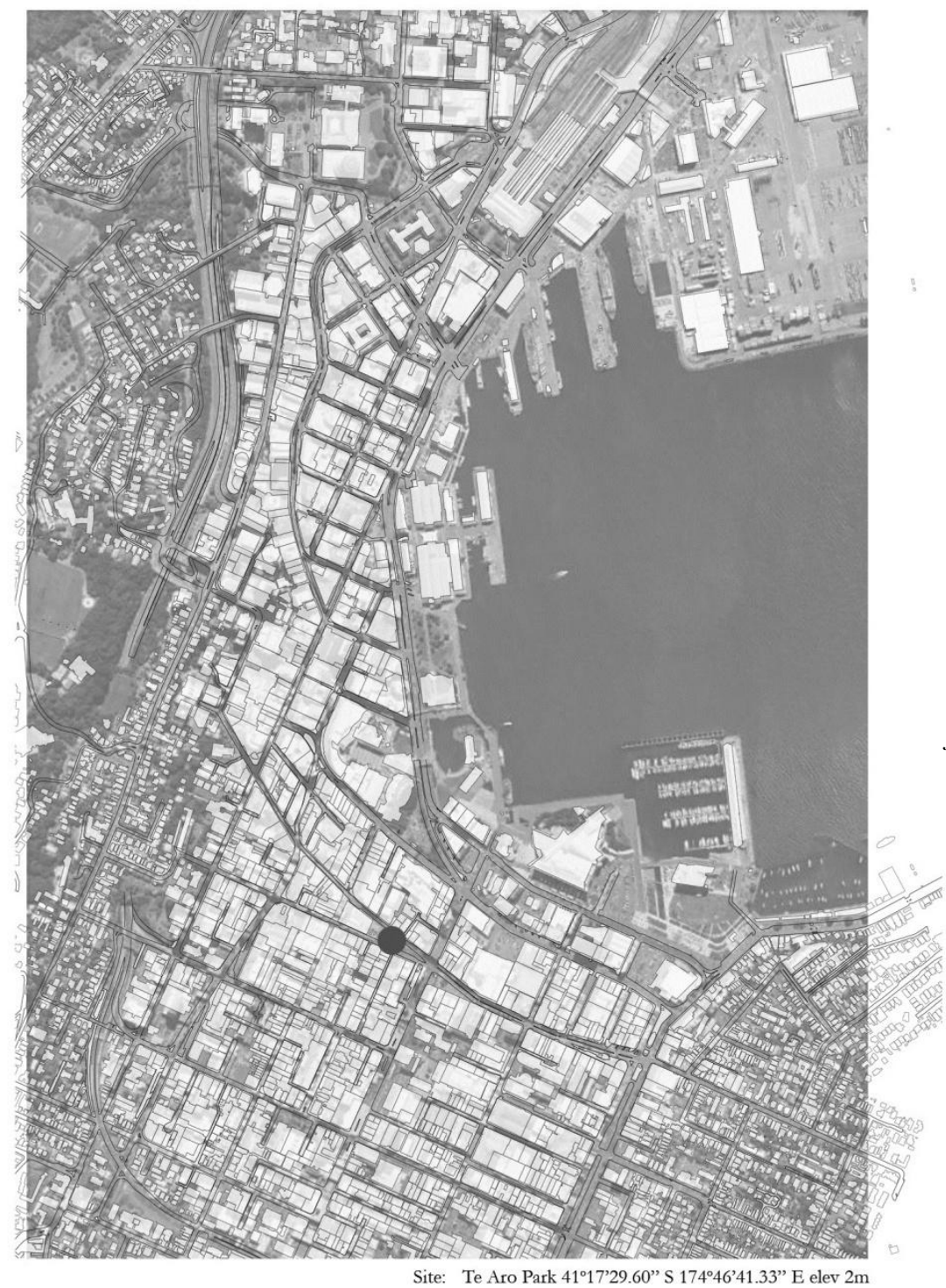

Fig 3.1] Diagrammatic mapping of identified site: Author adapted from, Google Earth.

This chapter introduces the design component of the thesis. The design is an architecture school that has been amalgamated with a park. The design responds to the criteria that was established in chapter three. Predicated on the research in chapter three the following is required to inform the programmatic and formal response.

1. Architecture must accommodate loitering.

2. Architecture must reject autonomy

3. Architecture must operate as an accessible marketplace.

In order to apply each strategy, this section of the thesis has utilised a specific site as a productive vehicle for design development. The selected site is Te Aro Park . Te Aro Park is located in Wellington which is the capital city of New Zealand. The site was selected because it is a genuinely public space that experiences a high density of pedestrian movement. The site that has been selected will enable the design component of the thesis to implement each strategy while it simultaneously addresses the issue of detachment. 

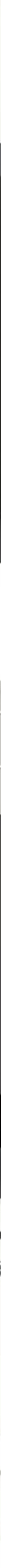


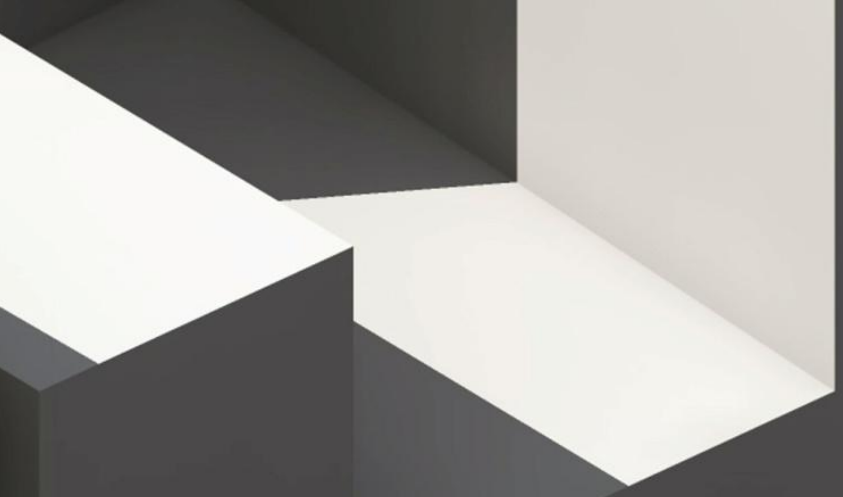




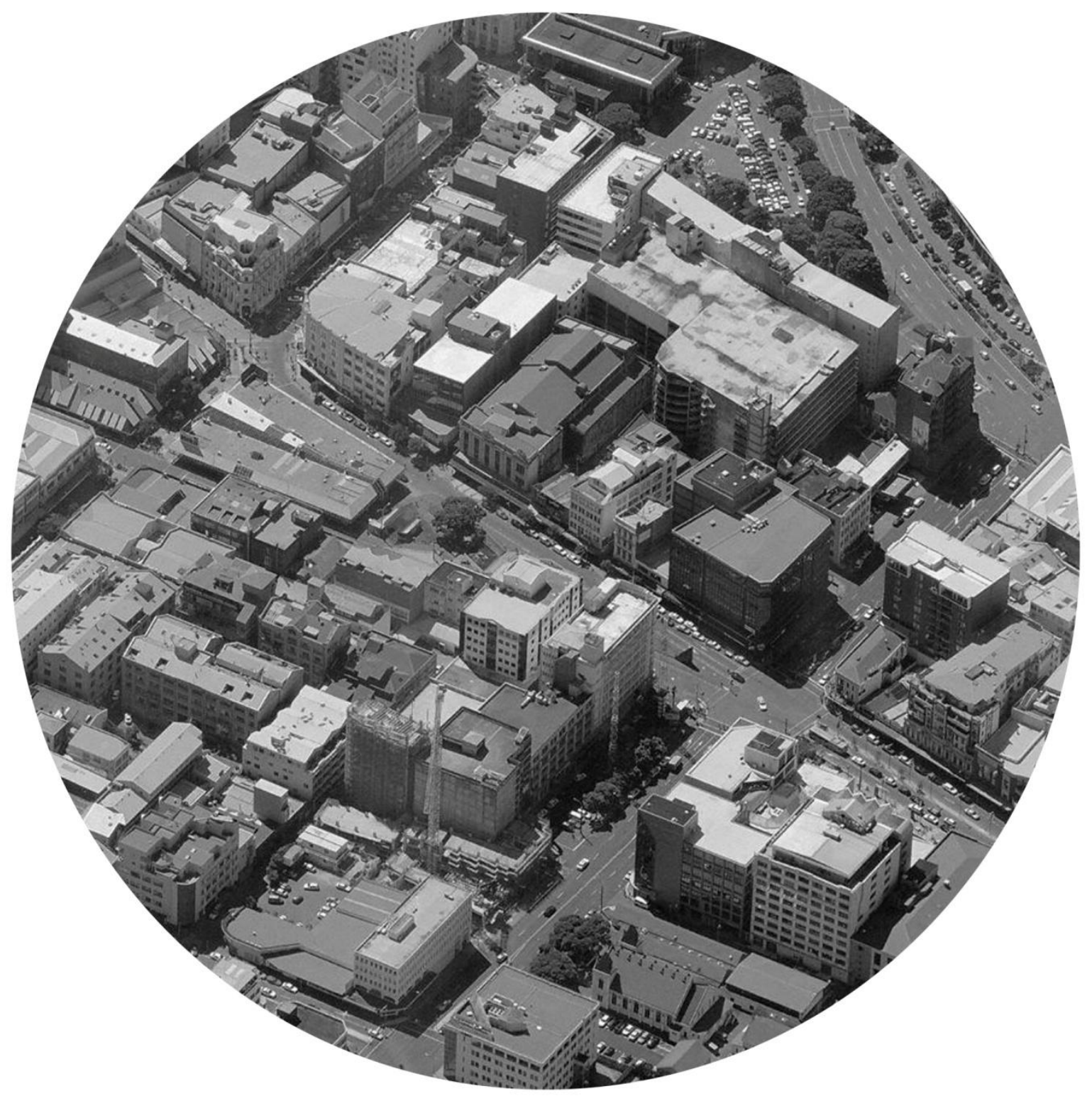

Fig 3.3] Axonometric of identified site: Author adapted from, Bing Maps. 

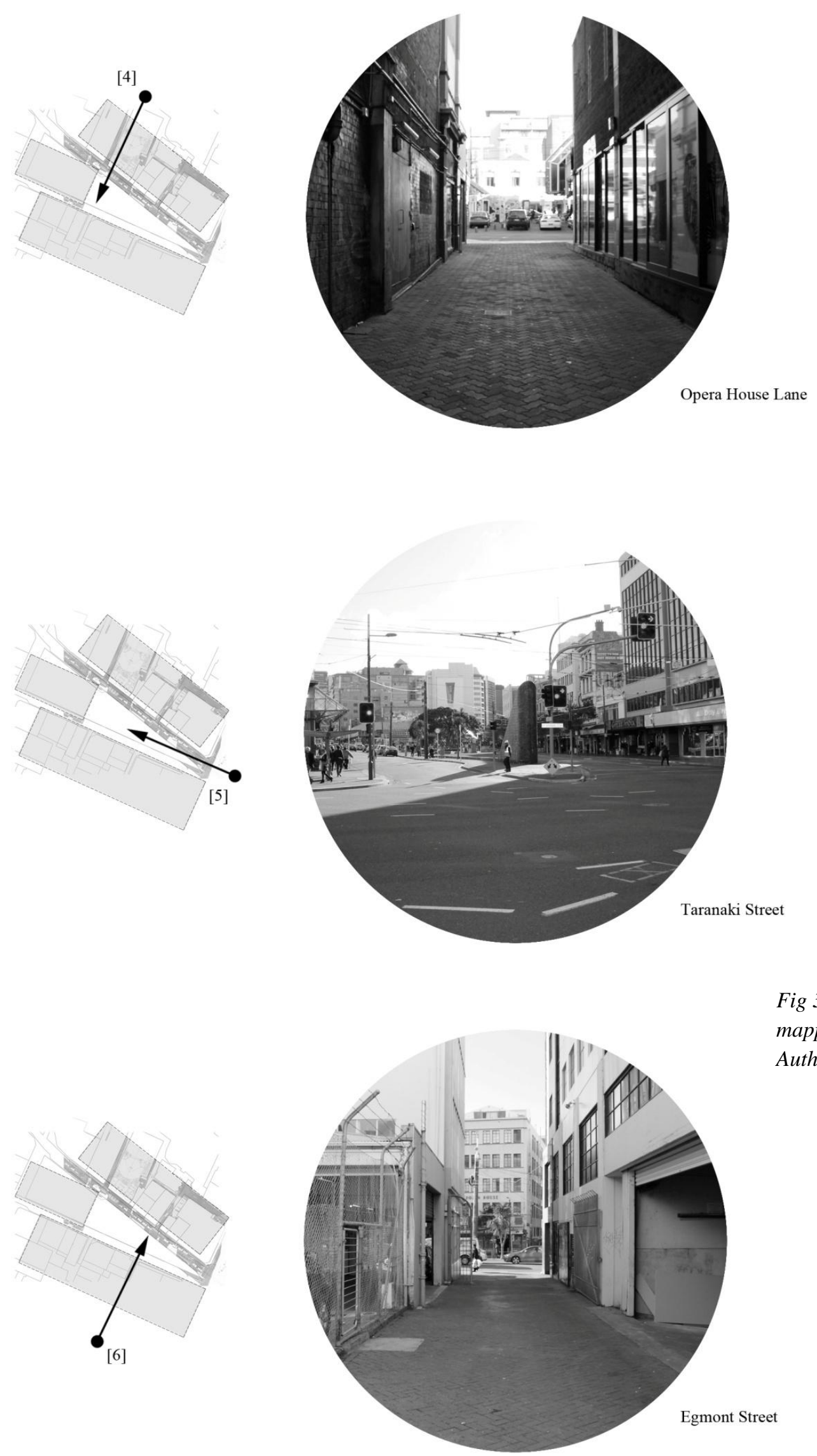

Fig 3.4] Diagrammatic mapping of identified site: Author's Collection 

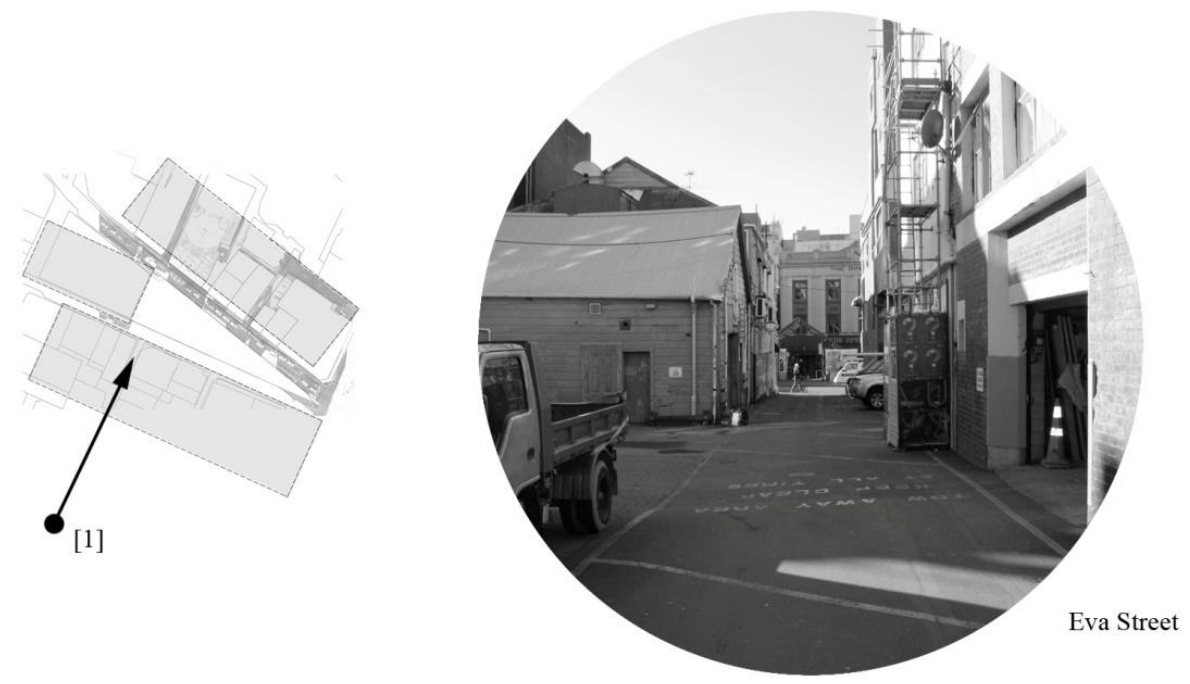

[2]
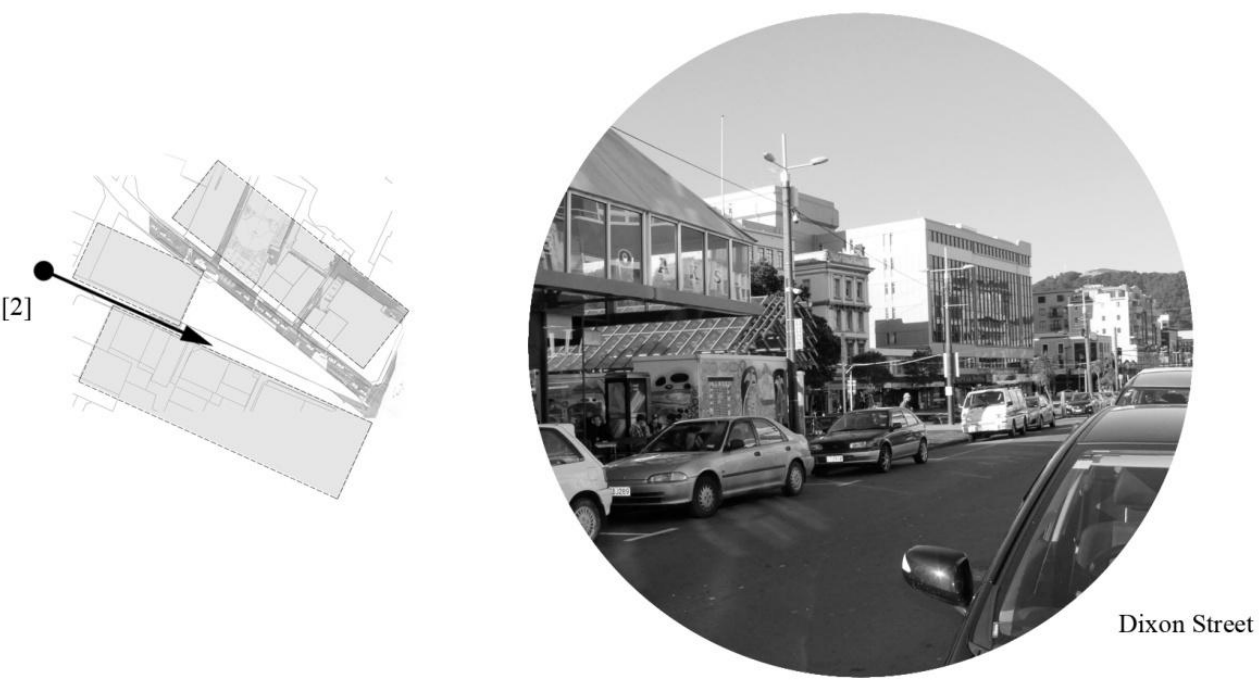

Fig 3.5] Diagrammatic mapping of identified site:

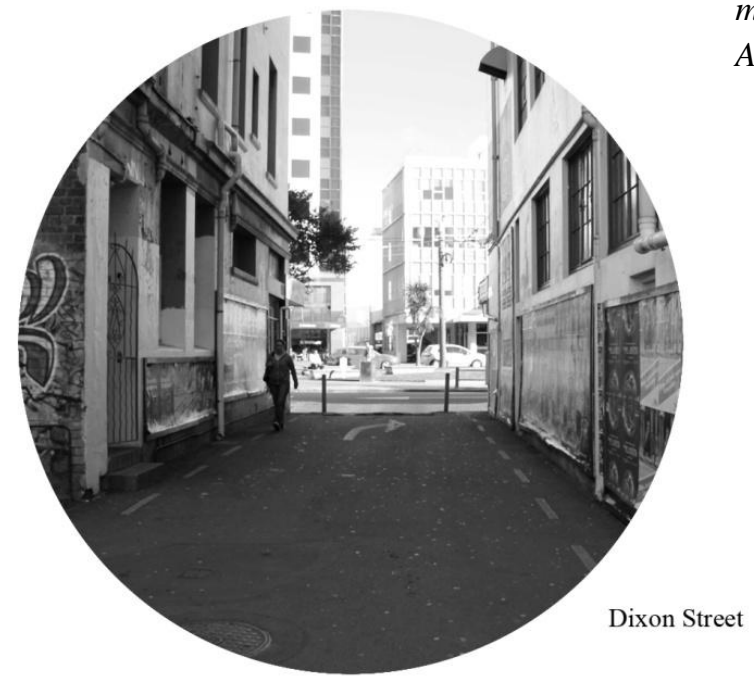
Author's Collection

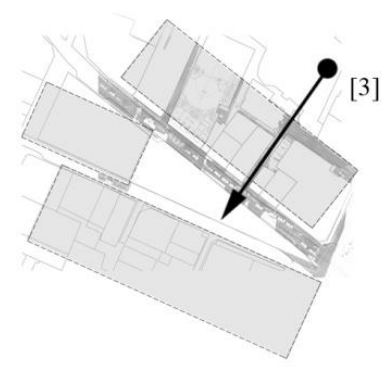




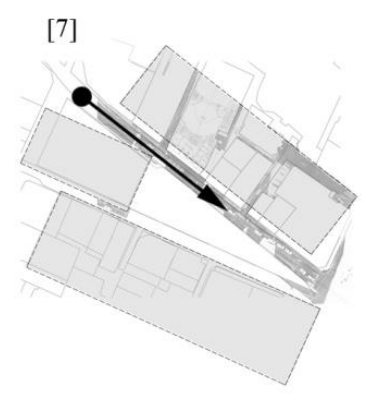

Fig 3.6] Diagrammatic mapping of identified site: Author's Collection

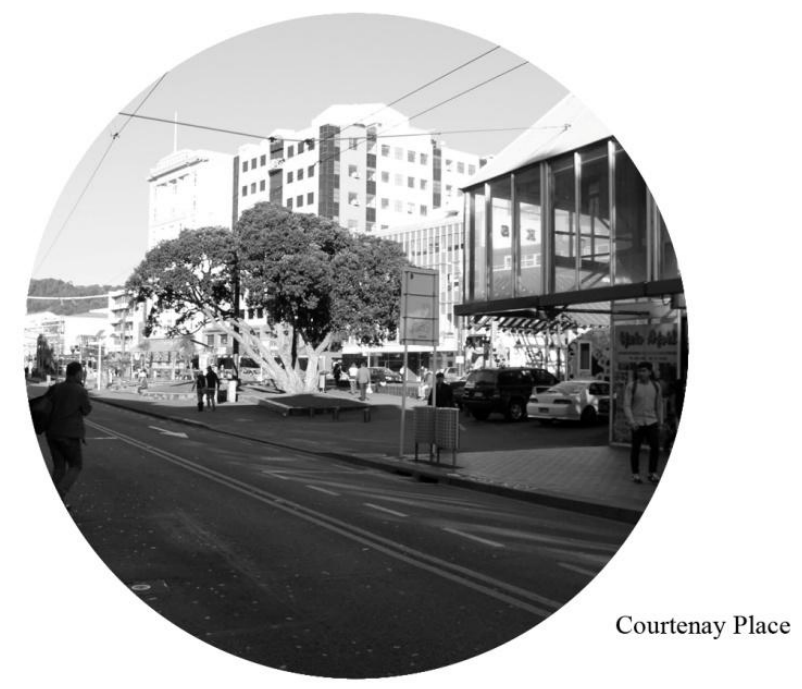

The thesis has established that an architecture school is isolated from the profession and the public. The problem occurred because physical segregation had secluded the building from public consciousness. In order for the design to accommodate public interaction it has been located in an area that experiences a high density of pedestrian circulation. If a site is subjected to a high density of pedestrian movement it will provide increased exposure. Increased exposure will enhance the opportunity for members of the public to initiate exchange and provide the opportunity for the public become active participants. Inhabitation of the park results in simultaneous inhabitation of the architecture school. The design does not require the public to initiate exchange because the design is the initiator. 


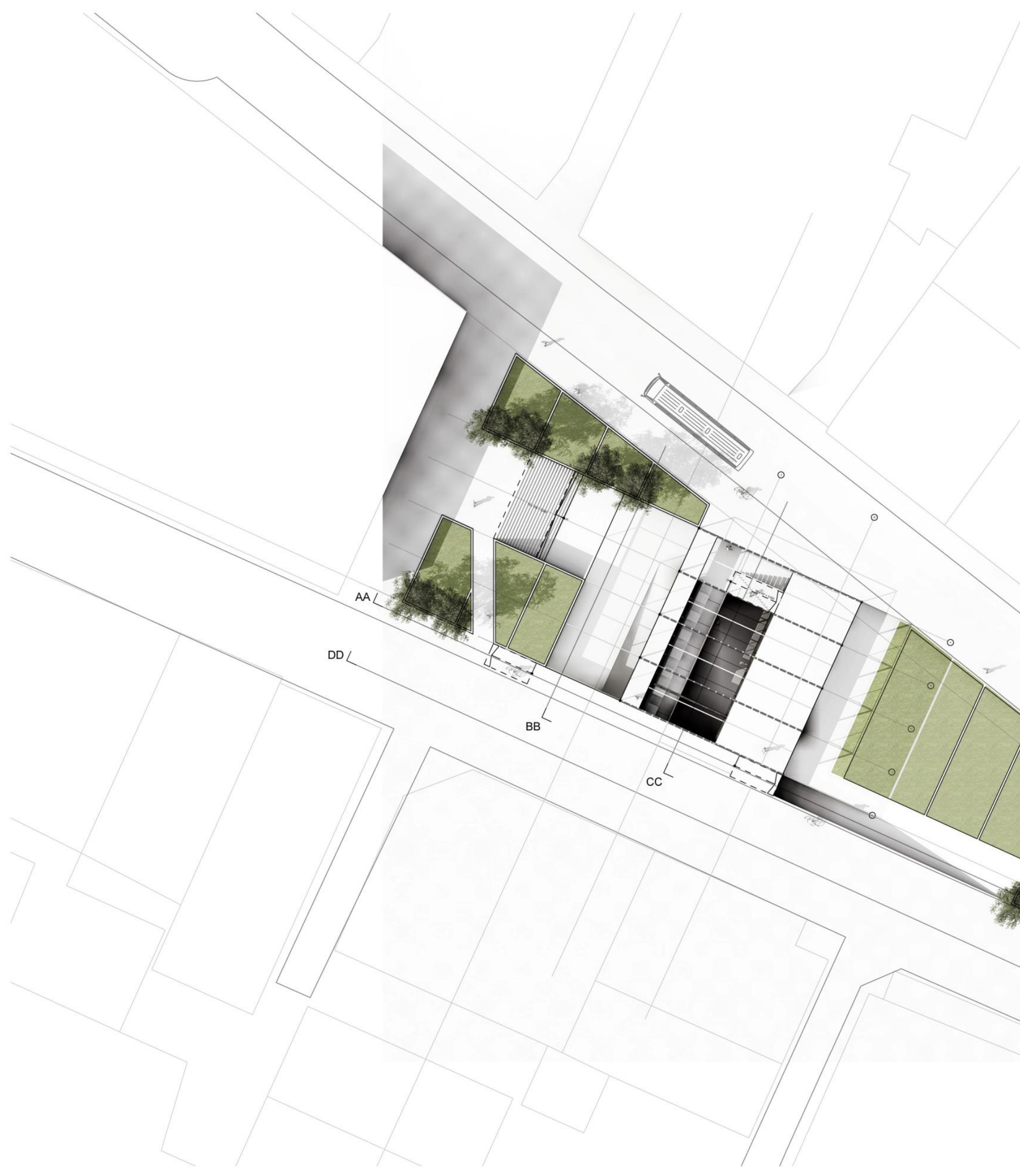

Fig 3.7] Site plan that

depicts the designed site:

Author's Collection 


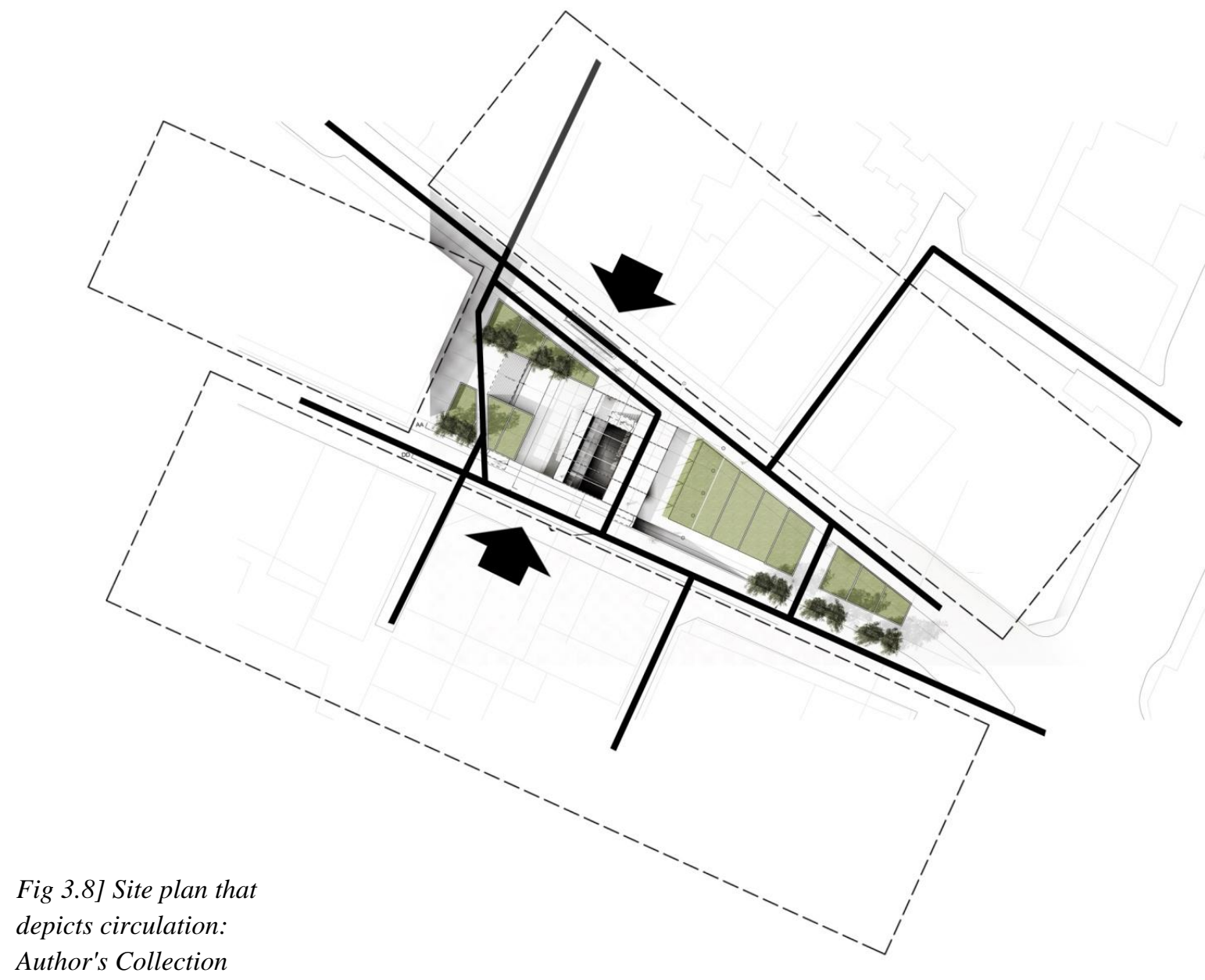

Site Analysis: Inversions of Privacy

[Architecture must accommodate loitering]

In order to destabilise the distinction between 'inside' and 'outside' and facilitate loitering the design is a continuation of the surrounding context. The intent of the diagram is to identify the opportunities for pedestrian circulation to engage the site and permit prominent connections between the interior and the exterior. The illustrated diagram identifies multiple penetration points. The numerous pedestrian routes that penetrate the site provided the opportunity to capitalise on the circulation strategy utilised in the Carpenter Centre, designed by Le Corbusier in 1963. The Carpenter Centre demonstrated that if a footpath is continued from the street into a building it can increase pedestrian access, integrate the building with the surrounding context and encourage prolonged loitering. The continuation of pedestrian circulation will enable the site to subliminally engage the public as opposed to requiring the public to initiate the engagement. 


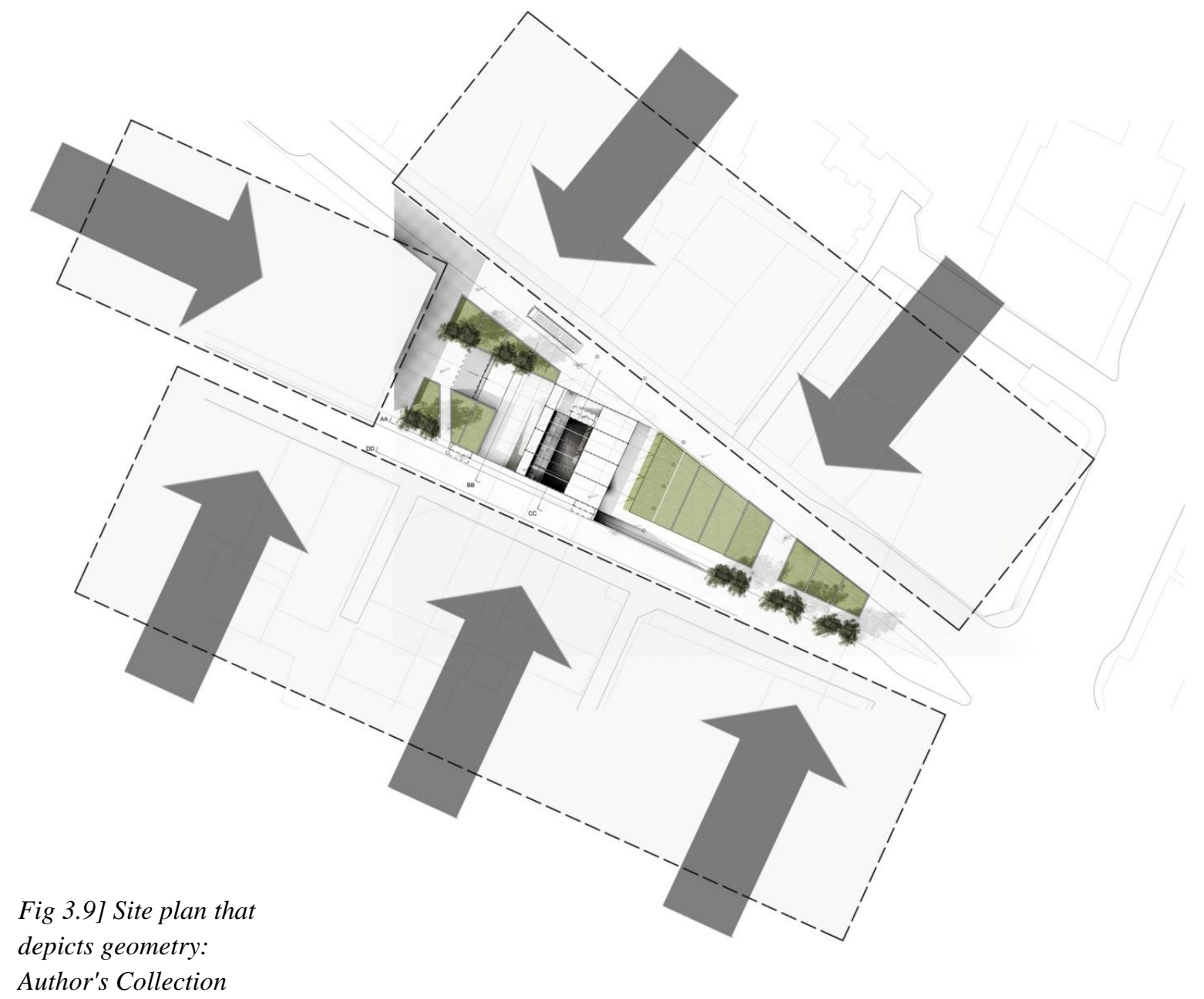

Site Analysis: Architecture as Art

[Architecture must reject autonomy]

In order to frame architecture as art and stimulate a broader public discourse the design has rejected autonomy and it operates as an interactive instillation. If architecture is permitted to operate as an interactive instillation it can shift the experience of the city that occurs in a state of distraction to a state that occurs through concentration. Architecture that is experienced in a state of concentration has the potential to stimulate selfreflection and critical thinking. The intent of the diagram is depict how the site has framed the design. The illustrated diagram has identified that the triangular site is situated between two rectangular grids. The surrounding buildings frame the selected site because they produce a visual disparity between solid [the buildings] and void [the site]. The surrounding buildings function in a similar manner to a picture frame. The selected site operates as the image contained within the frame. 


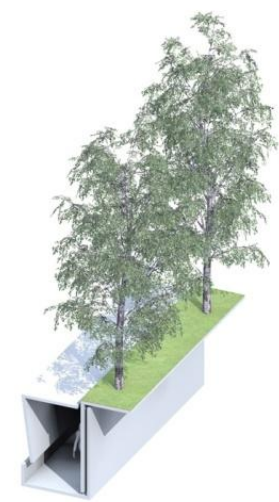

A

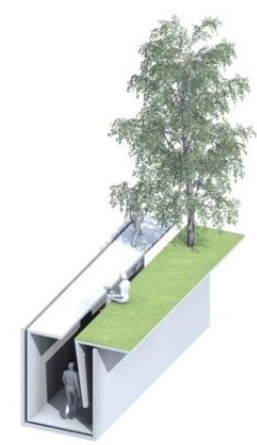

B

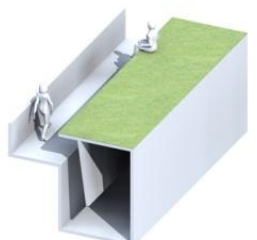

$\mathrm{C}$

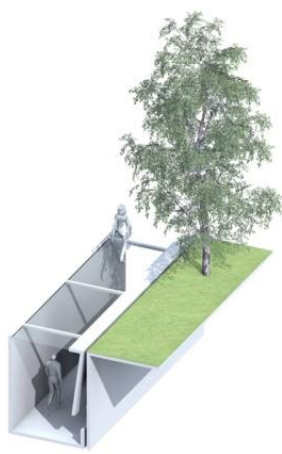

$\mathrm{D}$

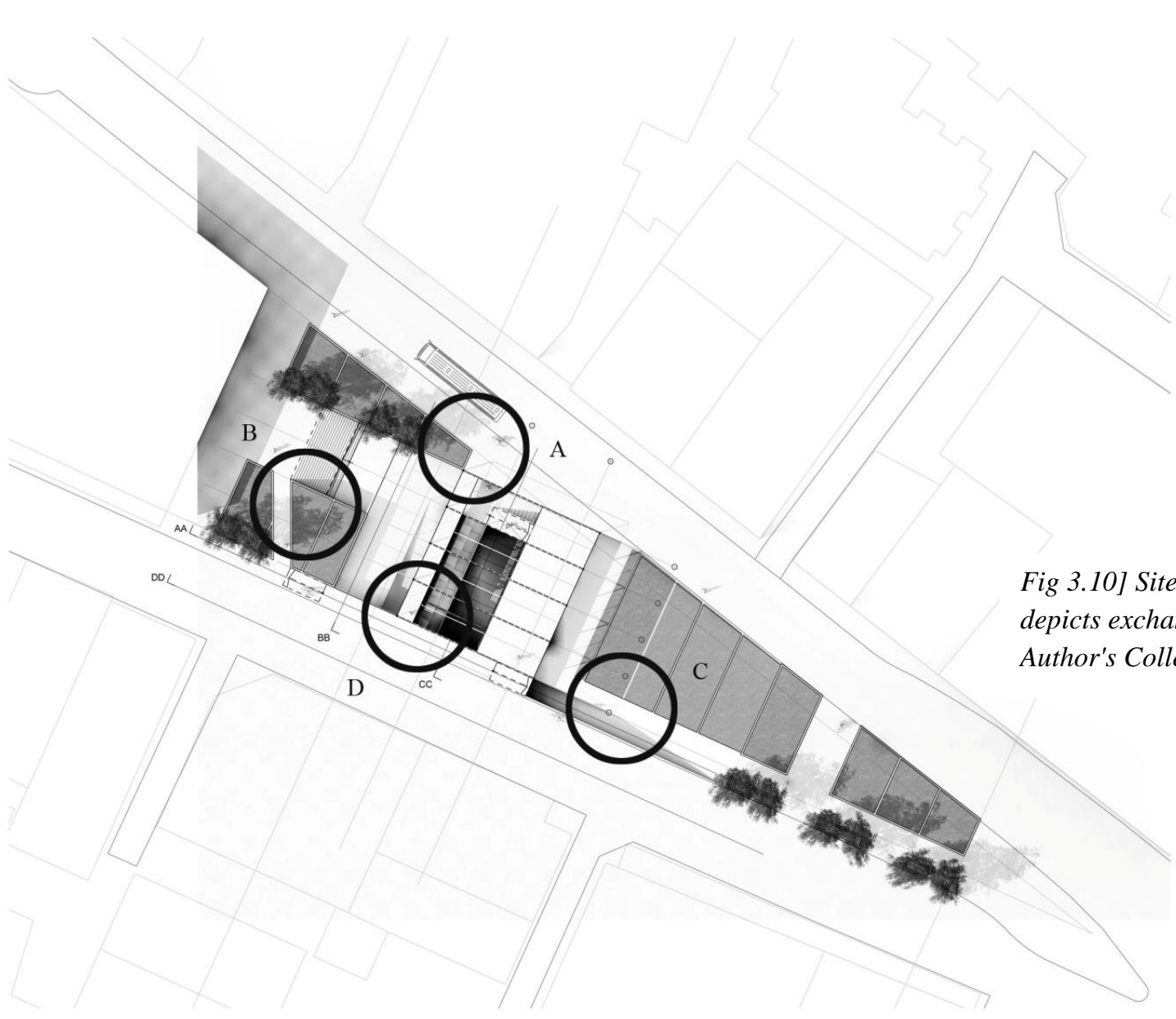

Site Analysis: University as Marketplace

[Architecture must operate as an accessible marketplace]

In order for an architecture school to operate as a marketplace the form and the surrounding context must be intertwined. Integration presents the opportunity to expose the interior and provide uninhibited access. The intent of the diagram is to identify the relationship between site visibility and pedestrian access. Site visibility and pedestrian access can be utilised as a productive tool to integrate the form and the surrounding context. The site contains multiple exchange points that permit disparate views. The exchange points permit and obstruct different aspects of the architecture school. In order for an architecture school to operate as an accessible marketplace it is required to be visually and physically accessible. 


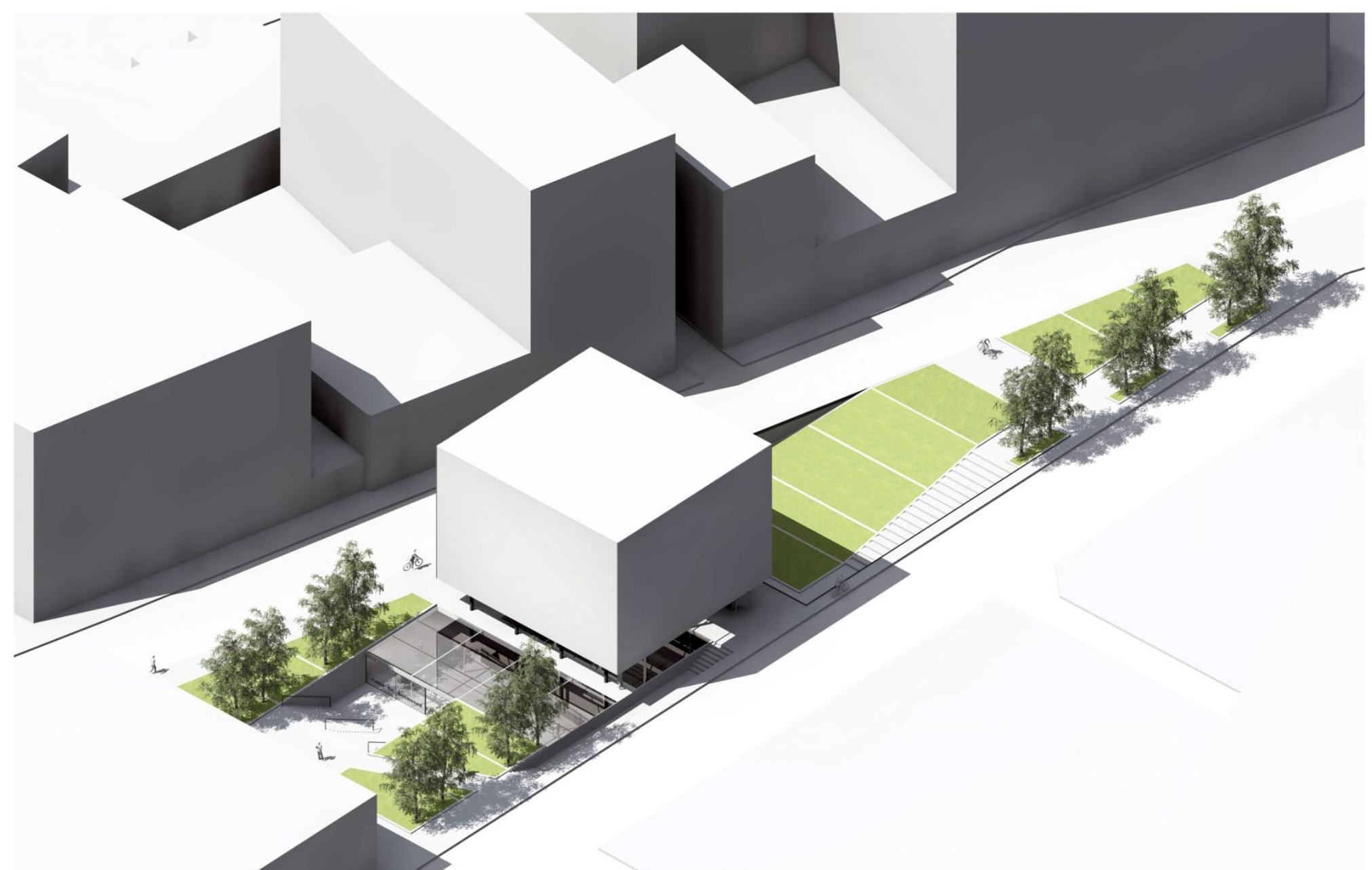

Fig 3.11] Site axonometric that depicts geometry: Author's Collection

(1.)

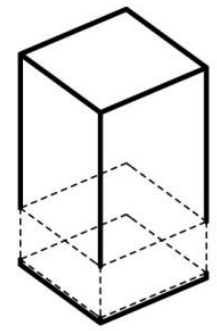

(2.)

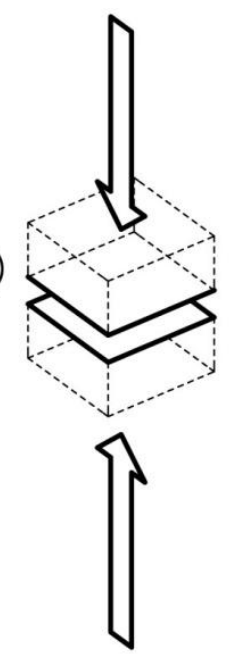

(3.)

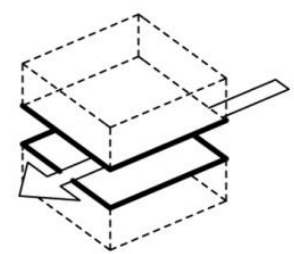

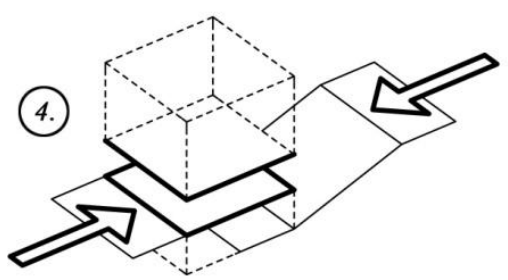

The large mass that is suspended in the site is utilised as a device to direct and frame an observers' vision toward the interior. 


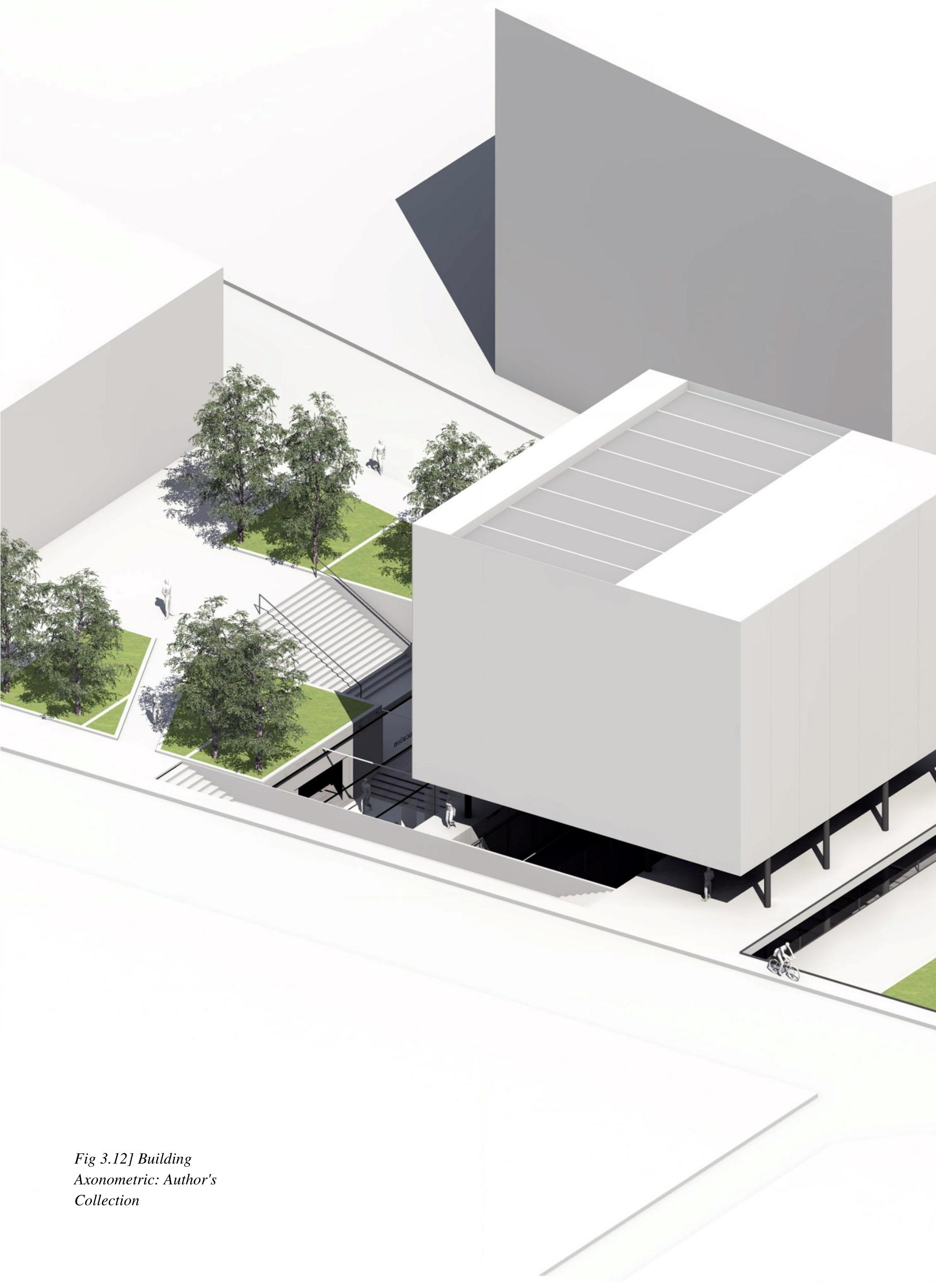





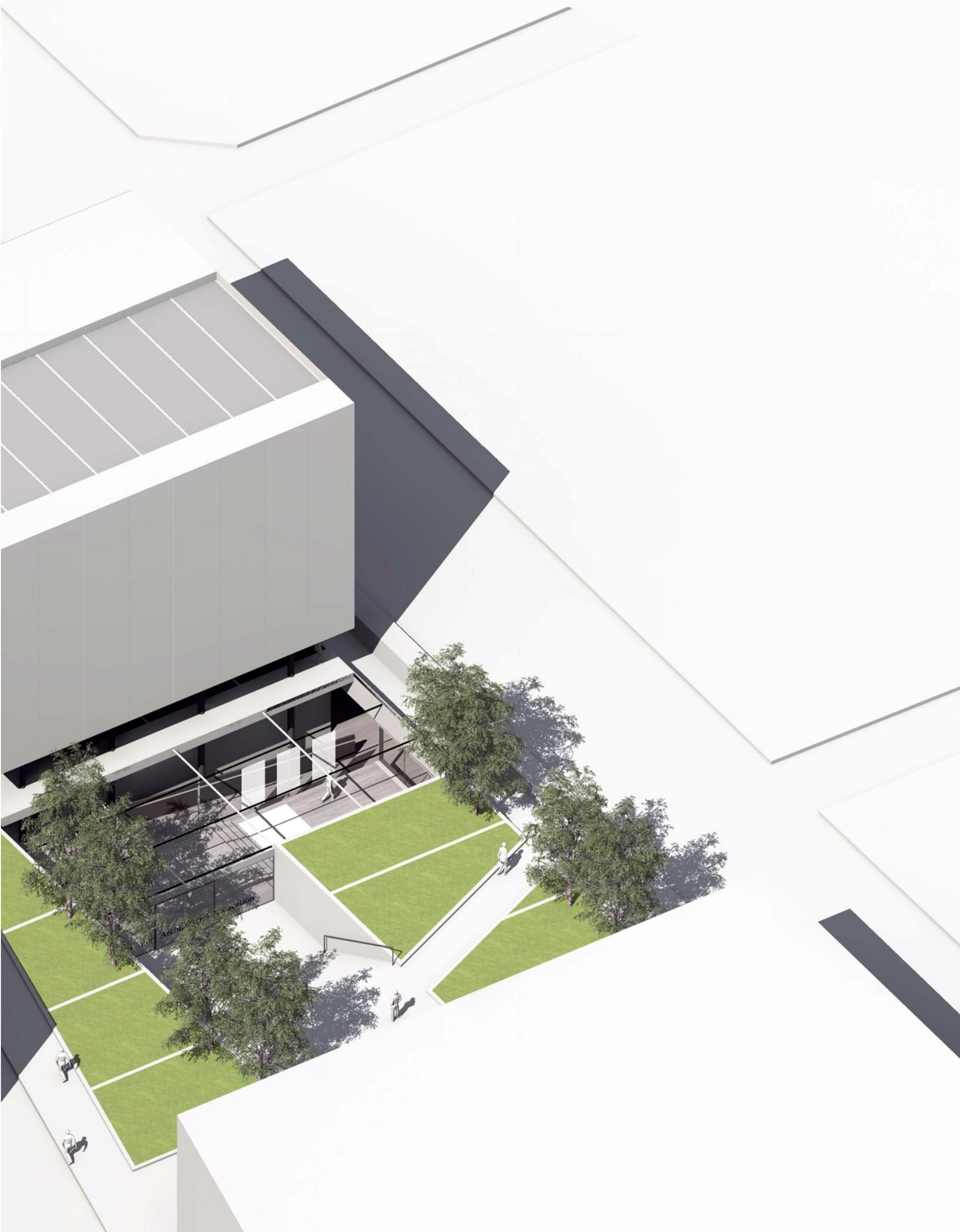


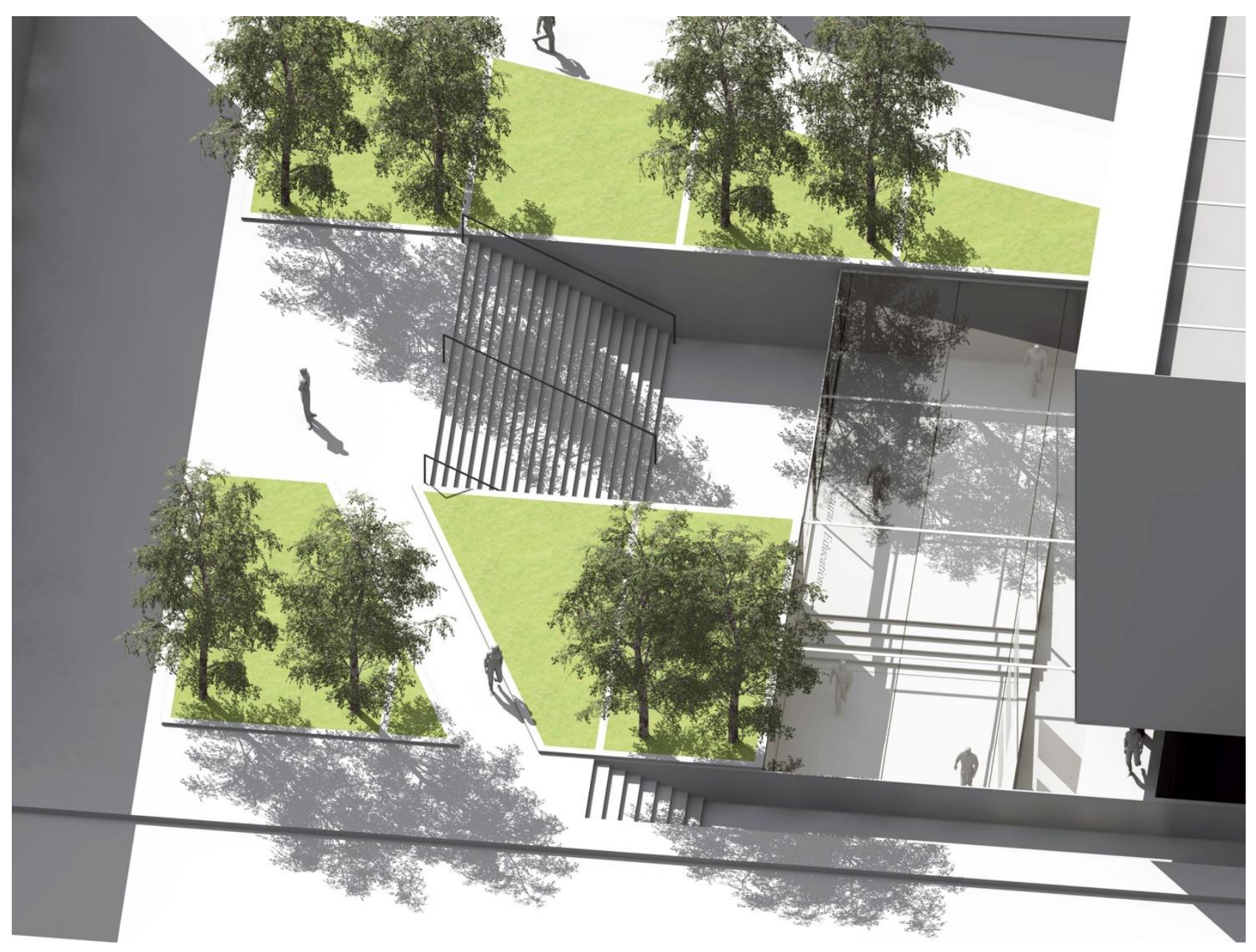

Fig 3.14] Site axonometric that depicts park and gallery space: Author's Collection

The thesis has established that the public feel as though they are trespassing when they inhabit an architecture school. In an attempt to dissolve this perception the internal facilities are composed around multiple passageways that penetrate the building. Each internal space is visually accessible from the park. An individual who inhabits the park is able to witness the internal activities that occur in the building. 


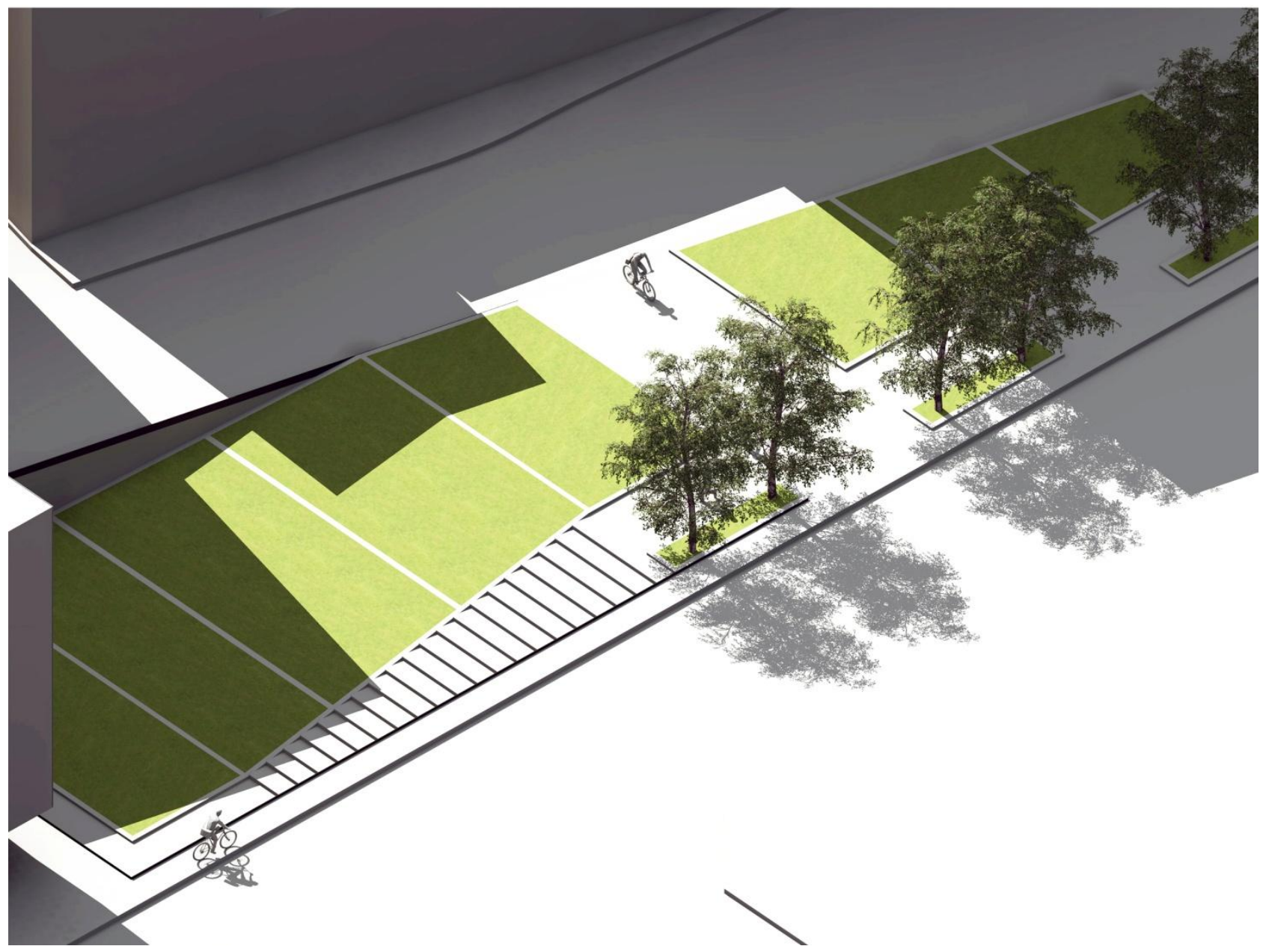

Fig 3.15] Site axonometric that depicts urban amphitheatre: Author's Collection

The park and the footpath descend into the building. The sloped surface produces an urban amphitheatre that directs an individual's attention toward the building. The large mass that is suspended in the centre of the design operates as a screen and enables architectural film to be projected onto it. The urban amphitheatre operates as a threshold that mediates between the city and the architecture school. 


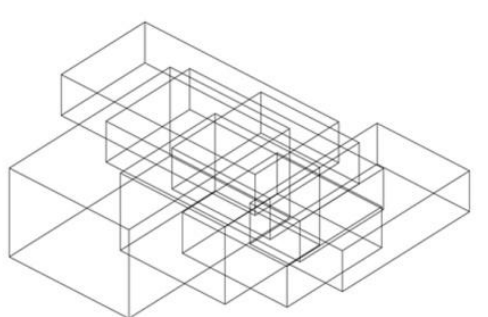

Conceptual Iteration - 001
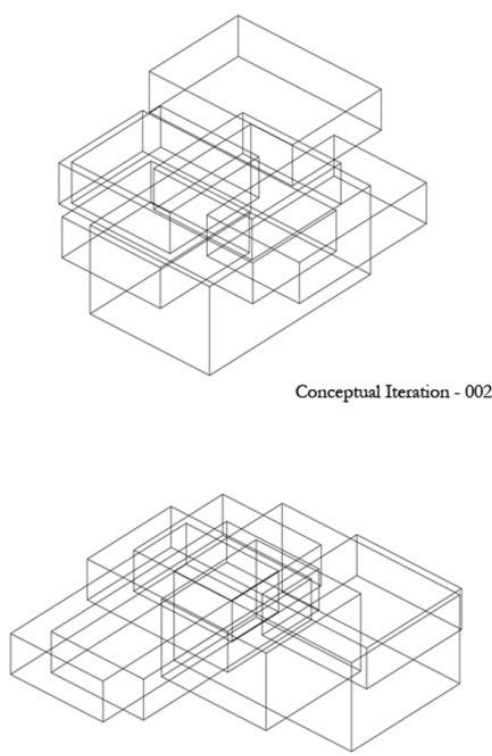

Conceptual Iteration - 003
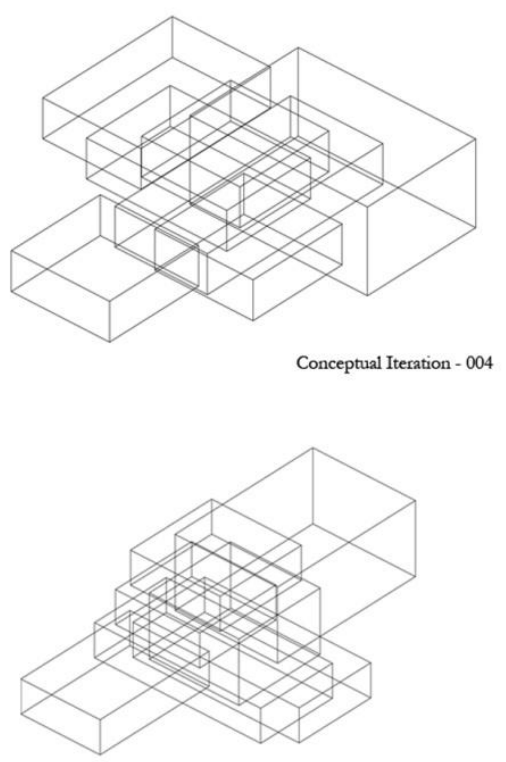

Conceptual Iteration - 005

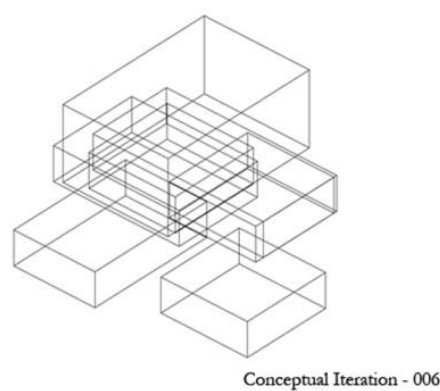

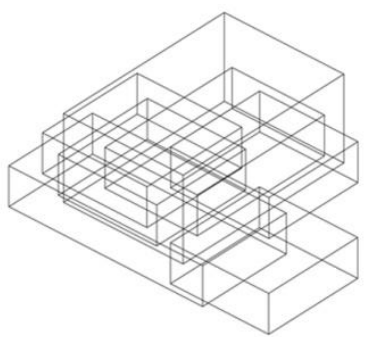

Conceptual Iteration - 007
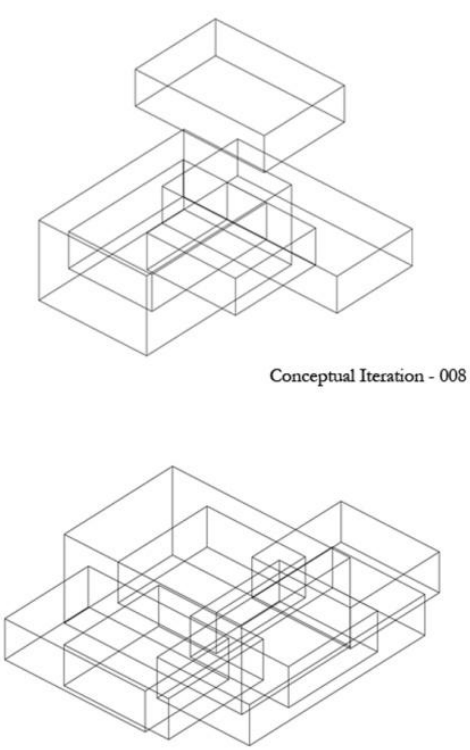

Conceptual Iteration - 009

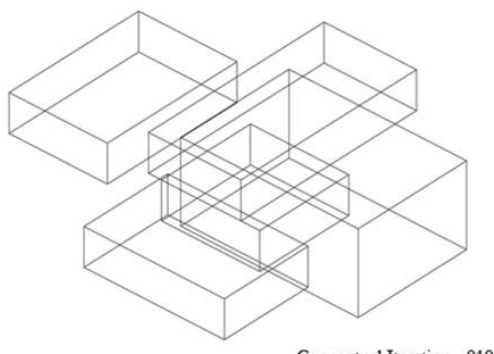

Conceptual Iteration - 010
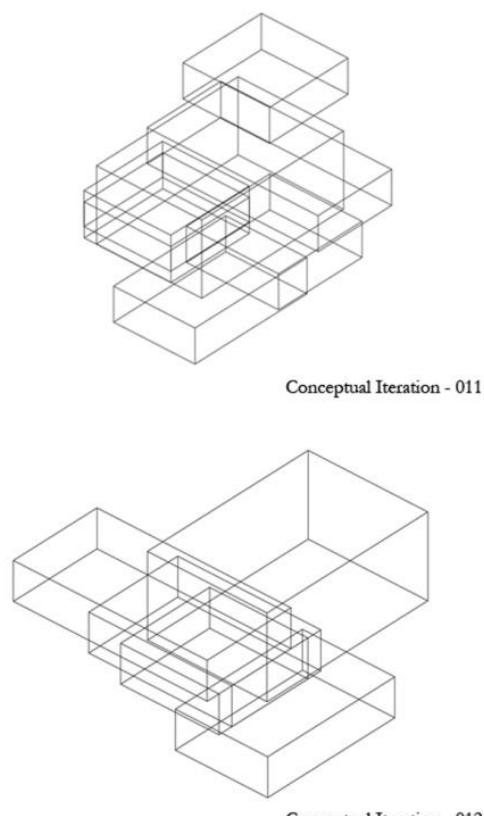

Fig 3.16] Diagrammatic mapping that depicts formal logic: Author's Collection 


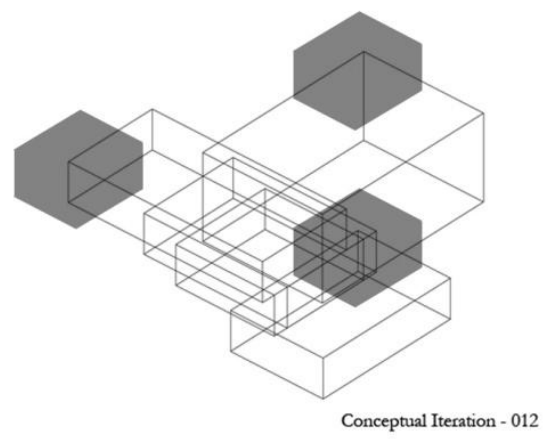

Fig 3.17] Diagrammatic mapping that depicts formal logic: Author's Collection

The design is conceived as an assemblage of components that have been integrated into the existing context. 


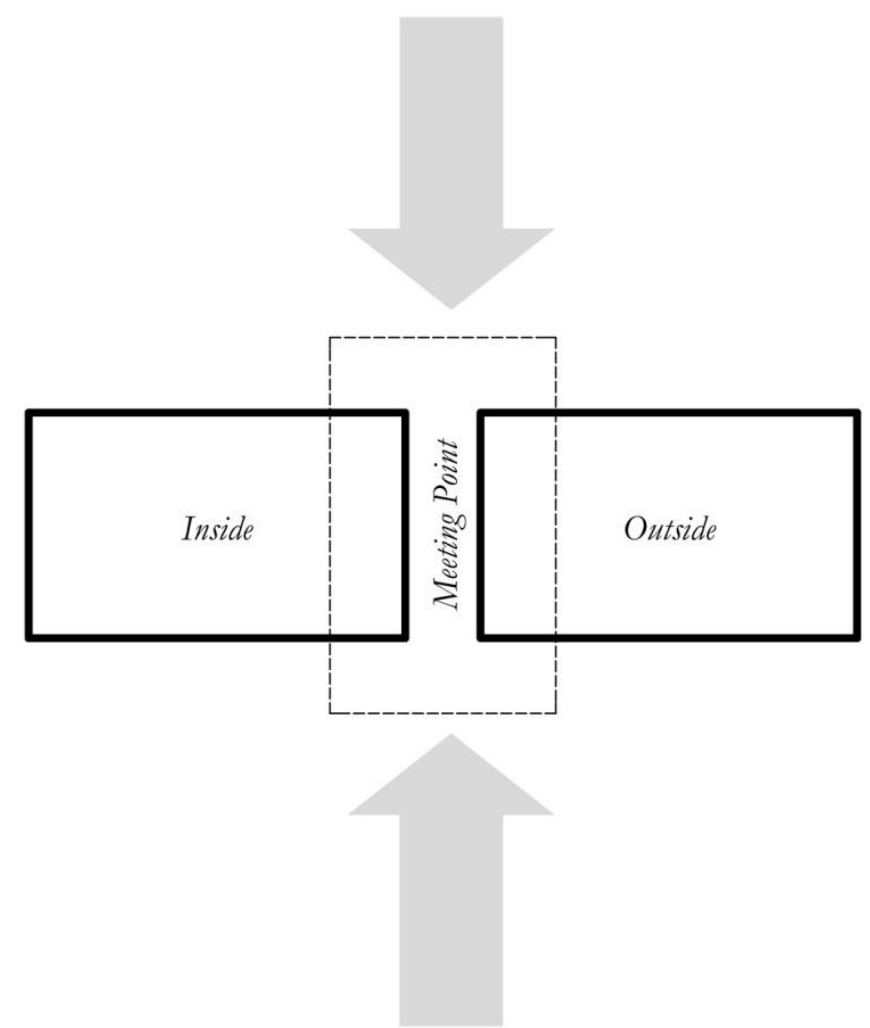

Fig 3.18] Building diagram that depicts formal logic: Author's Collection

The design is a catalyst that amalgamates the inside and the outside. 


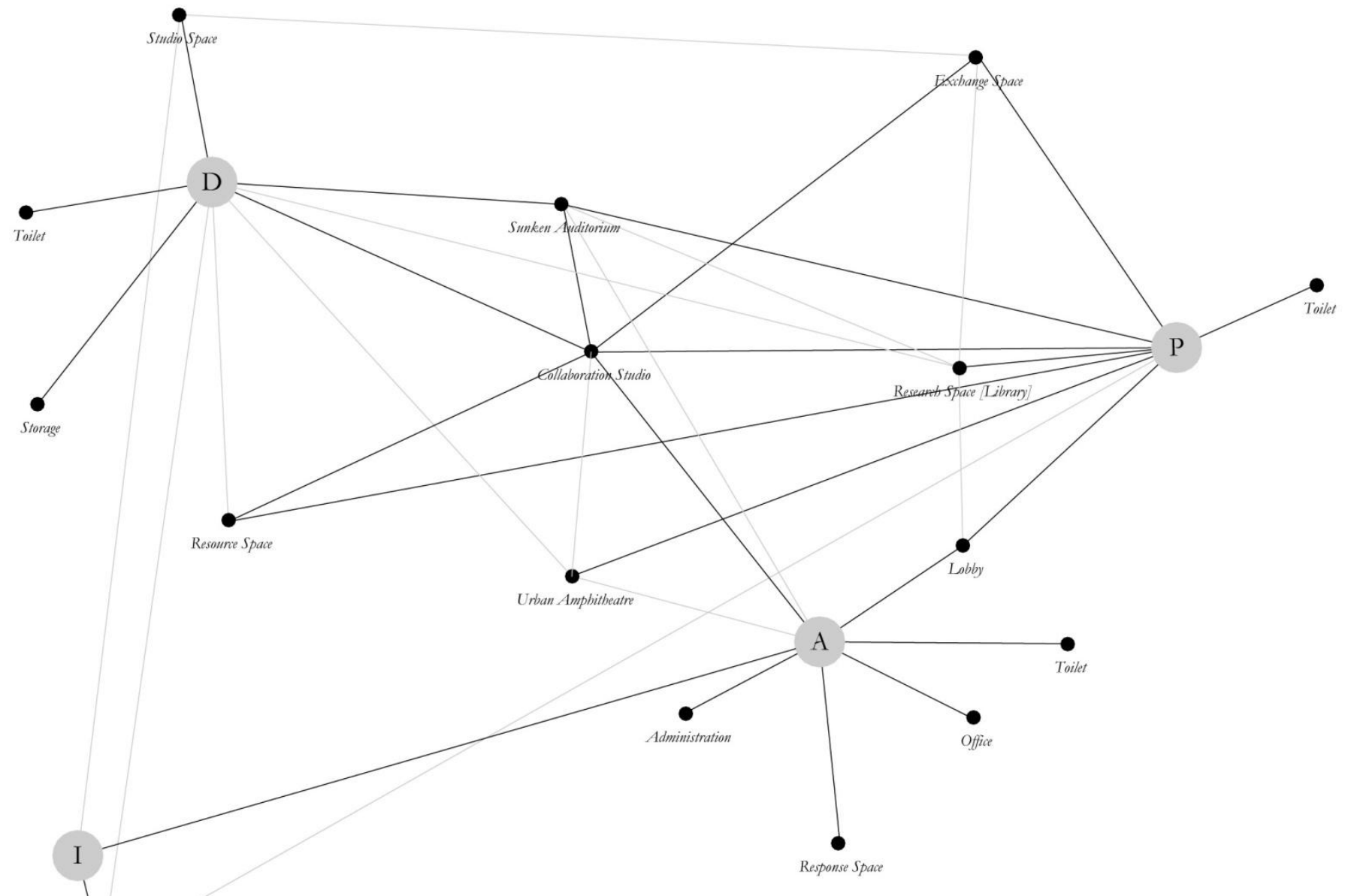

P Public Space

Fig 3.19] Diagrammatic mapping that depicts program composition:

A Administration Author's Collection

D Design Studio

I Indeterminate Space 

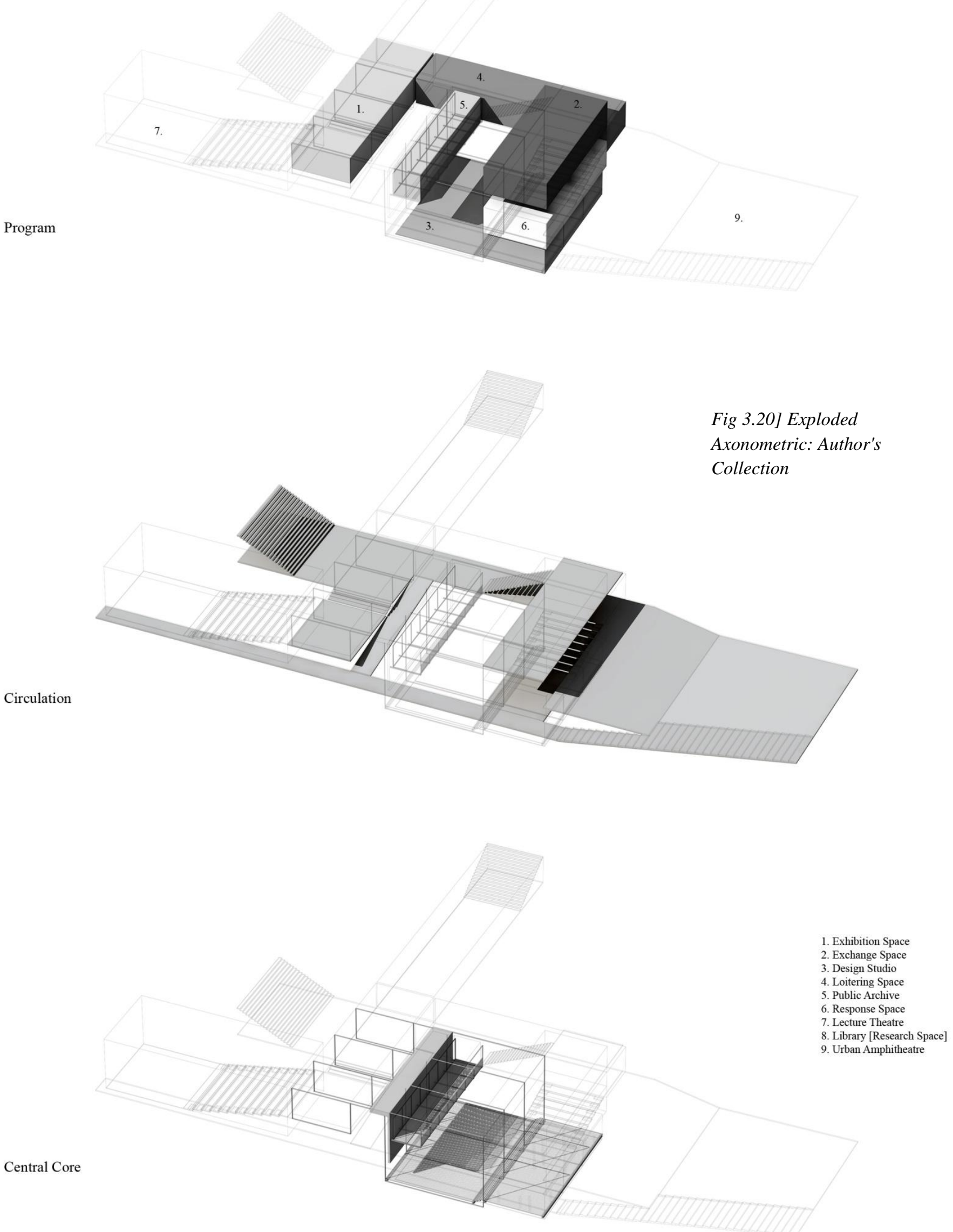

Fig 3.20] Exploded

Axonometric: Author's

Collection

Loitering Space

5. Public Archive

Lecture Theatre

Library [Research Space]

Urban Amphitheatre 


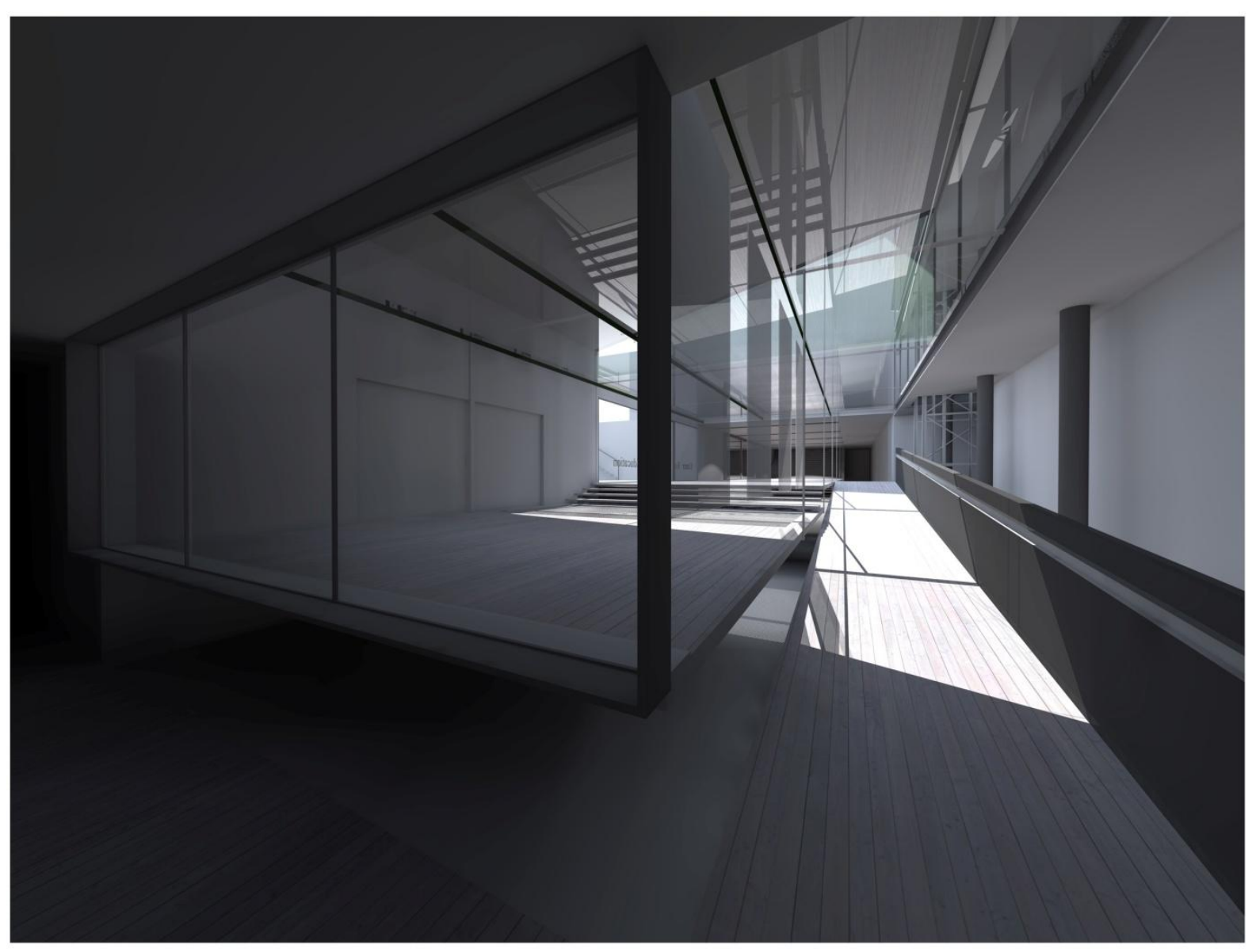

Fig 3.21] Gallery Space: Author's Collection 
The architectural facilities utilised by the students are contained within the central core. The central core functions as an exhibition space that exhibits the activities of the students and the output that they produce.

The large mass that is situated in the centre of the design is intended to stimulate public interest. The large mass frames an individual's attention toward the centre of the site.

The park penetrates the interior of the design and it provides circulation between individual spaces.

Fig 3.22] Section: Author's

\section{Collection}
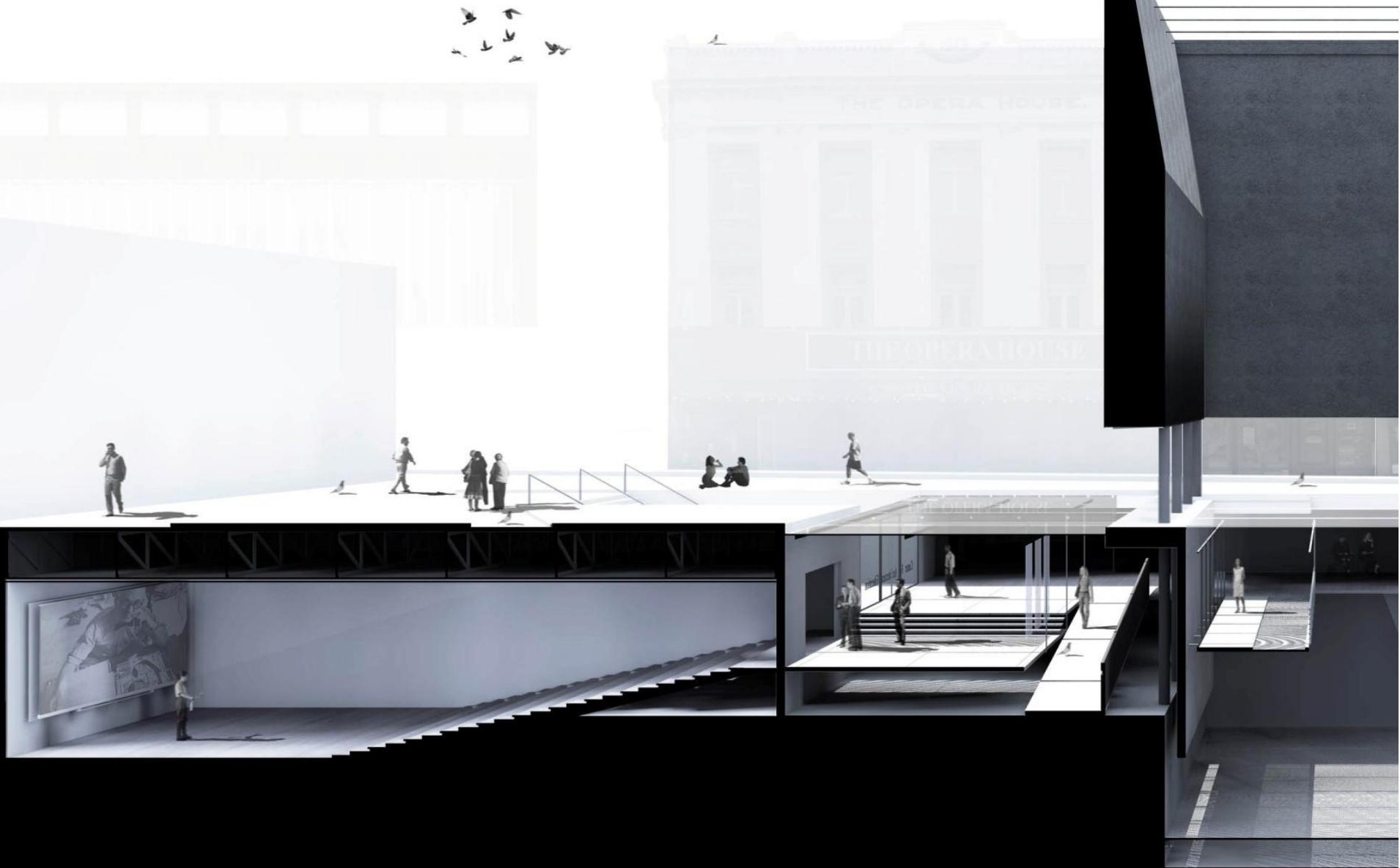

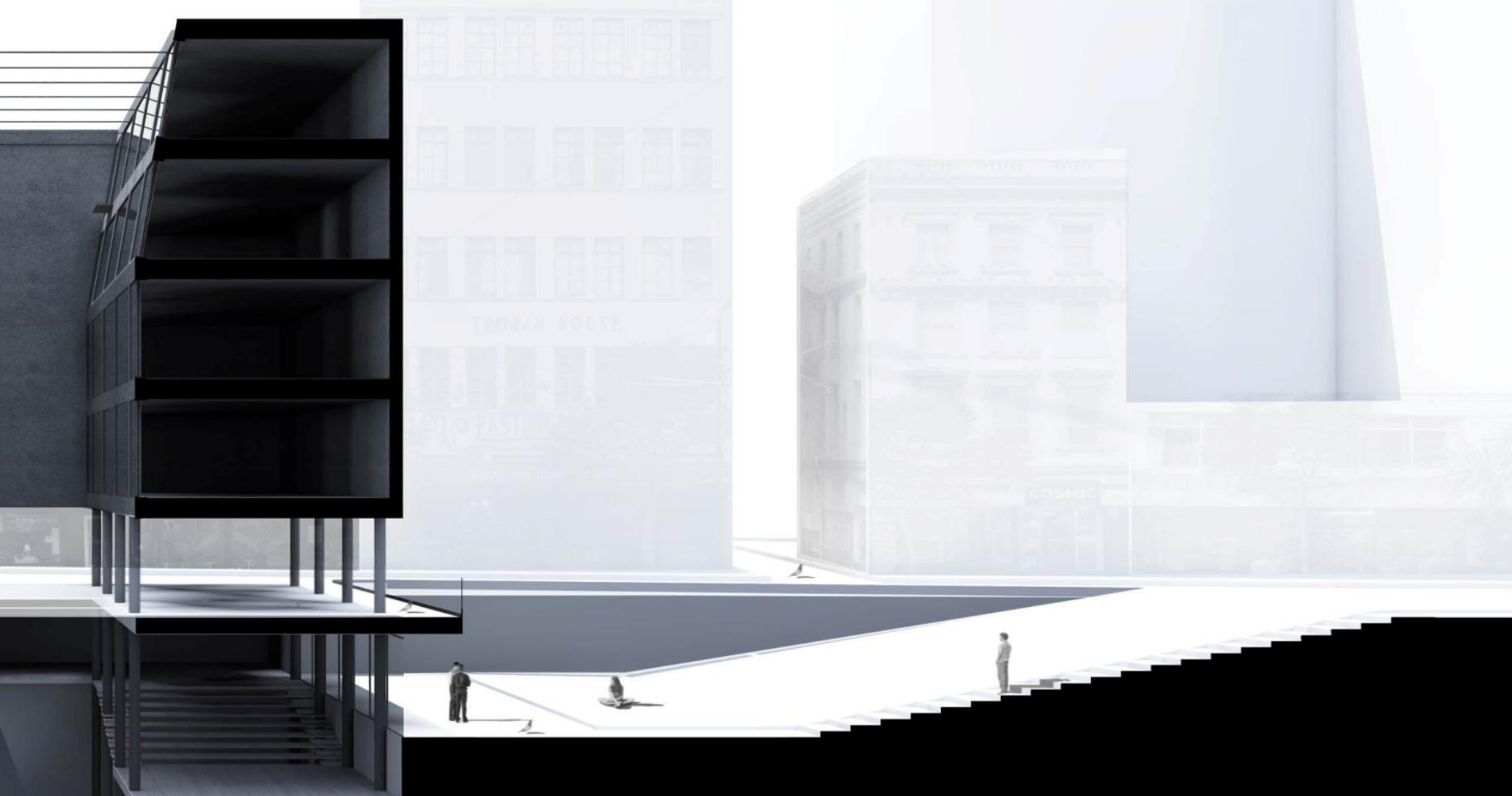
Fig 3.23] Ground Floor:

Author's Collection 



$$
\text { 似 }
$$



Fig 3.25] Section: Author's

Collection 


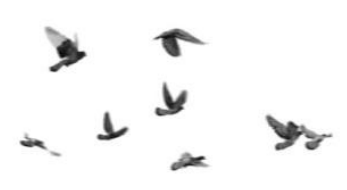

1 


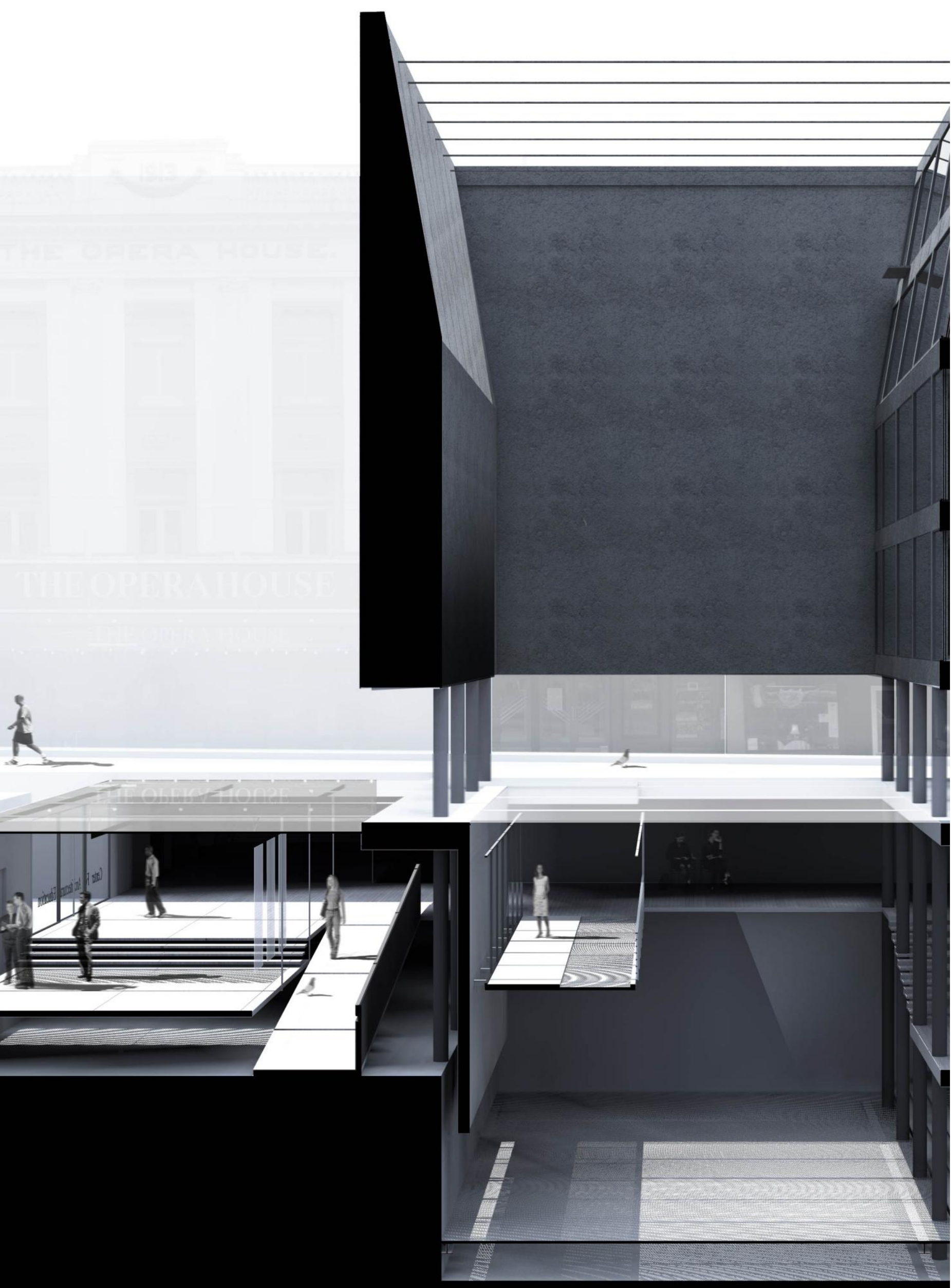



Fig 3.26] Circulation

Perspective: Author's

\section{Collection}

01 Exchange

02 Viewing Gallery

03 Staircase

04 Display Units

05 Thoroughfare

01

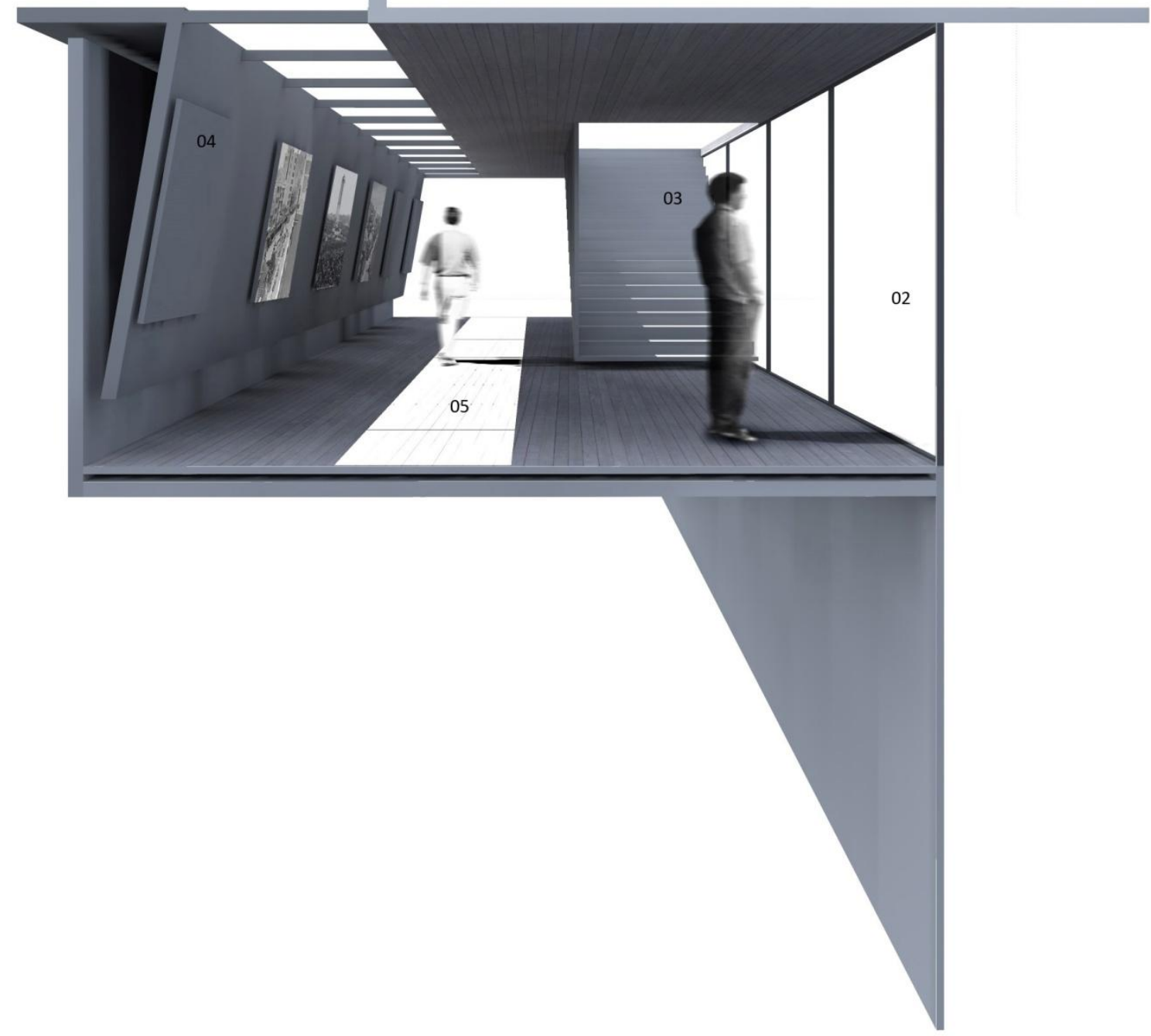




\section{[4]}

\section{Critical Appraisal}

\subsection{Architecture as (a) Medium (of communication).}

"The point of view of modern architecture is never fixed, as in baroque architecture, or as in the model of vision of the camera obscura, but always in motion, as in film or in the city. ${ }^{5 "}$

\subsection{Architecture is a medium of communication.}

The intent of the critical appraisal is to identify the applicability of the proposed solution and discuss how the design operated as a medium. In order to establish how the design itself is a medium of communication and consequently how that medium operates, the thesis utilises the Shannon-Weaver model of information theory. The Shannon-Weaver model of information theory is the archetypal model of transmissive communication. This model reduces communication to a process of 'transmitting information'. ${ }^{6}$ The model states that all communication consists of five components arranged in a linear format. The components are: an information source, transmitter, noise, receiver and destination. ${ }^{7}$ The chapter has been divided into six sections. Each section examines an individual component in the Shannon-Weaver model of information theory.

\subsection{Information Source:}

[The producer of the architecture is the information source].

Shannon and Weaver propose that communication is initially stimulated by an information source. ${ }^{8}$ The information source produces a message by encoding information into a transmittable form. ${ }^{9}$ In architecture, information is transmitted through the physical form. The individual who determines the physical form operates as the information source.

In contrast to other forms of media output the origin of the information source is not limited to a singular author. In the text 'Occupying Architecture', Jonathan Hill establishes that there are two occupations of architecture: the activities of the architect and the actions of the user. ${ }^{10}$
The architect produces architecture through design and the user produces architecture through use.

The architect and the user are not mutually exclusive information sources; they are interrelated and inform, and are informed by each other. Jonathan Hill establishes that a user can function as an (illegal) architect that constructs the space around them through design and use. ${ }^{11}$ The identification that architecture is subject to multiple authorship is important because it is the author that determines the physical form, and it is that form that operates as the transmission channel.

In the design there are three information sources. The building is the primary information source. The students and the public are the secondary information sources. The building transmits information through its formal composition. The students and the public transmit information through inhabitation. The students and the public operate as information sources because their actions in the building affect how others perceive the space.

\subsection{Message:}

[The message is derived from the specific manner in which the building has been intentionally assembled].

Shannon and Weaver propose that the content that has been transmitted from one end of the model to the other is the message. ${ }^{12}$ In architecture, the medium is the message. ${ }^{13}$ The message is derived from the specific manner in which the building has been intentionally assembled.

\subsection{Transmitter:}

[The architectural components transmit the message].

The transmitter is a component within the Shannon-Weaver model of information theory that transmits a message to a desired destination. The transmitter itself is able to operate as part of the message, or alternatively, it is the message. ${ }^{14}$ In architecture, it is the architectural components that transmit and construct the message.

In "Function and Sign: Semiotics of Architecture", Eco claims that Architecture uses codified objects to communicate functions. ${ }^{15}$ The communication is rhetorical because it persuades an individual to inhabit and perceive architecture in a specific manner. ${ }^{16}$ For example, the components of Gothic 


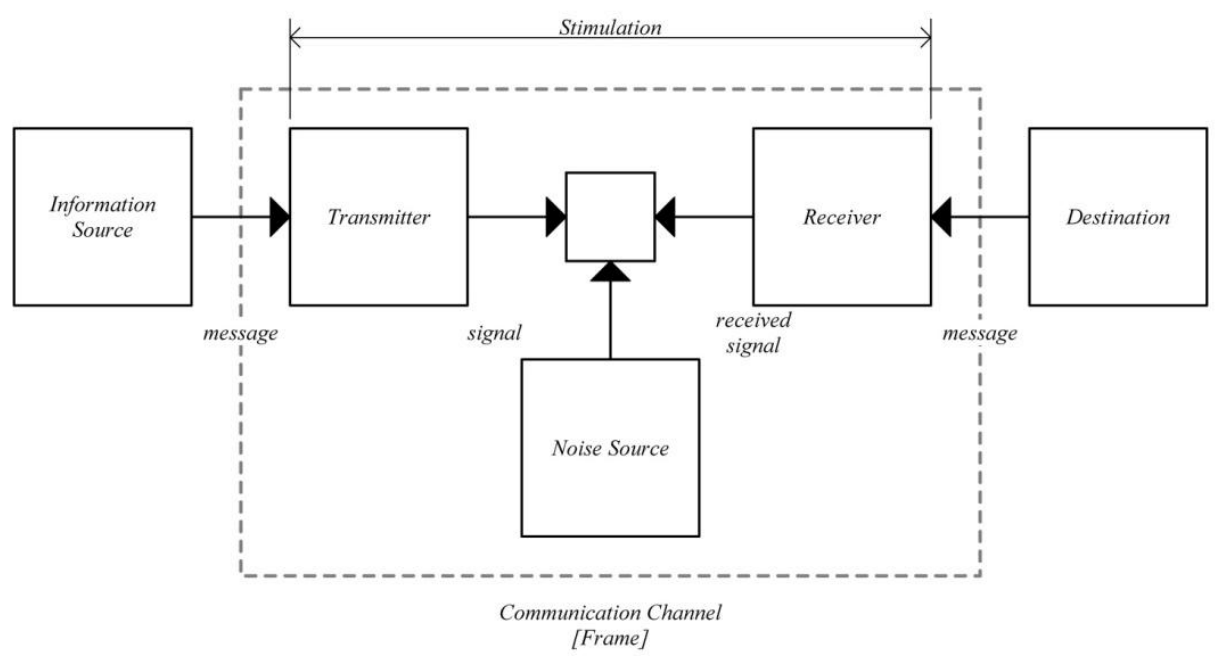

Fig 4.1] Communication Diagram: Author adapted from, Shannon-Weaver model of information theory.

The diagram provides a pictorial representation that identifies how discussion is stimulated and how discourse is framed. 
architecture (vaulted ceiling, pointed arches and the manipulation of scale in relation to the human body) communicated a pictorial reading of God's power. The pictorial reading that was communicated prompted submissive inhabitation. The spatial arrangement of the Gothic Cathedral served a communicative function: it communicated the act of submission to be fulfilled.

Architectural components transmit through program and codification. However, the amalgamation of those elements can, in addition, facilitate the transmission of impermanent imagery. That is, the actions of the user can be visually transmitted through architecture. ${ }^{17}$ This is evident in the text 'Beatriz Colonina - Privacy and Publicity', in which it is identified that architecture, television and film utilise similar modes of communication.

Colomina proposes that the modern transformation of architecture produces a space defined by walls of moving images. ${ }^{18}$ In contrast to predeceasing forms of architecture that were constructed from solid walls punctuated by small windows, modern architecture is constructed from lines of glass. It is the view and not the architecture that defines the space.

Therefore, the experience of modern architecture is similar to television and film because the viewer witnesses a series of successive events. The viewer is not able to arrest the image because it is under a constant state of flux. Colomina is informed through the German philosopher Benjamin Walters (1892-9140) and reinforces the notion that no sooner has an individual's eye grasped a scene than it has already changed. ${ }^{19}$

For example, an inhabitant within a modern shopping complex experiences the external environment through a glass facade. The events that occur outside of the building are received by the inhabitant as a series of impermanent imagery. The extent of their vision is determined by the frame itself. The architecture determines what is visually permitted and what is restricted.

In this context it is not the codification of individual elements that communicate; rather, the lines of glass function as a projection device enabling a message to be transmitted.

The text is important because it operates two-fold: first, it expands our understanding of architectural transmission and reception to include the projection of impermanent imagery; second, it reinforces the notion that architecture is not the sole authority of a single author. The transmission of impermanent imagery is produced by the actions of the user and enabled by the actions of the architect.

The message is transmitted through an architectural object(s) and is stimulated through function, codification and imagery.

\subsection{Noise Source:}

[Environmental and contextual obstacles that impact the perception of the receiver].

A generic depiction of 'noise' is defined as the distortion in communication transmission. ${ }^{20}$ In architecture, there are two forms of noise: channel noise and semantic noise. ${ }^{21}$ Channel noise is the environmental and contextual obstacles that impact the perception of the receiver. Semantic noise is the distortion that occurs as a result of individual understanding and expectations. Both forms of noise distort the communication transmission.

In a typical architecture school, semantic noise has resulted in an architecture school being perceived as a private space. In order to prevent this from occurring channel noise was utilised as a productive vehicle for design development. The selected site was a genuinely public space that experienced a high density of public engagement. The design was conceived as an assemblage of components that were integrated into the park. Inhabitation of the park resulted in simultaneous inhabitation of the architecture school. The spatial conditions that are prevalent in the park are translated into the building. The environmental and contextual obstacles in the site framed the design as a public space.

\subsection{Receiver:}

[The manner in which the message is received is determined by the individual's subjective expectations].

Shannon and Weaver propose that the decoction process is the inverse of the encoding process. The decoder interprets the initial message in order to understand the content. In architecture, the information source produces architecture and the decoder interprets that architecture. 


\subsection{Destination:}

[The destination is the occupant who inhabits the architecture].

In order for communication to be completed a receiver is required at the end of the channel that the information source used. ${ }^{22}$ The receiver is an individual whom the sender had intentionally or inadvertently transmitted a message to. In architecture, the receiver is the inhabitant. ${ }^{23}$

\subsection{Conclusion:}

In contrast to the pictorial representation of architecture and printed discourse, this thesis examined how architecture itself can operate as a medium of communication. The thesis has identified that an architecture school has the capacity to contribute to public discourse through the visual affects of form making and the social implications of that same form.

5 Colomina, Beatriz. Privacy and Publicity: Modern Architecture As Mass Media. Cambridge, Mass MIT Press, 2000. Print. Pg. 6

6 Nöth, Winfried. Handbook of Semiotics. Bloomington [u.a.: Indiana Univ. Press, 1998. Print. Pg.174

7 Nöth, Winfried. Semiotics of the Media: State of the Art, Projects, and Perspectives. Berlin [u.a.: Mouton de Gruyter, 1997. Print. P.690

$8 \quad$ Ibid. P.690

9 Nöth, Winfried. Semiotics of the Media: State of the Art, Projects, and Perspectives. Berlin [u.a.: Mouton de Gruyter, 1997. Print. P.690

10 Hill, Jonathan. Occupying Architecture: Between the Architect and the User. London: Routledge, 1998. Print. P.20

11 Ibid. P.20

12 Nöth, Winfried. Handbook of Semiotics. Bloomington [u.a.: Indiana Univ. Press, 1998. Print. Pg.174

13 McLuhan, Marshall. Understanding Media: The Extensions of Man. Routledge [u.a., 2006. Print. P.32

14 Colomina, Beatriz. Privacy and Publicity: Modern Architecture As Mass Media. Cambridge, Mass MIT Press, 2000. Print. P.78

15 Leach, Neil. Rethinking Architecture: A

Reader in Cultural Theory. New York: Routledge, 1997. Print. Pg.56 1997. Print. P.119

17 Colomina, Beatriz. Privacy and Publicity: Modern Architecture As Mass Media. Cambridge, Mass MIT Press, 2000. Print. P.34

18 Colomina, Beatriz. Privacy and Publicity: Modern Architecture As Mass Media. Cambridge, Mass MIT Press, 2000. Print. P.65

19 Colomina, Beatriz. Privacy and Publicity: Modern Architecture As Mass Media. Cambridge, Mass MIT Press, 2000. Print. P.76

20 Narula, Uma. Communication Models.

New Delhi, India: Atlantic, 2006. Print. P.27

$21 \quad$ Ibid. P.27

$22 \quad$ Ibid. P.27

23 Ibid. P.27 


\section{Bibliography}

Alexander, Christopher. A Pattern Language: Towns, Buildings, Construction. New York: Oxford Univ. Press, 1979.

Colomina, Beatriz. Privacy and Publicity: Modern Architecture As Mass Media. Cambridge, Mass MIT Press, 2000 .

Gans, Deborah, and Corbusier Le. The Lecorbusier Guide. New York: Princeton Architectural Press, 2000.

Gargiani, Roberto. Rem Koolhaas, Oma: The Construction of Merveilles. Lausanne: EPFL Press, 2008.

Giedion, Sigfried. Space, Time and Architecture: The Growth of a New Tradition. Cambridge, Mass, 1974.

Hertzberger, Herman, and Herman Hertzberger. Lessons for Students in Architecture: 2. Rotterdam: Press, 2000.

Hill, Jonathan. Occupying Architecture: Between the Architect and the User. London: Routledge, 1998.

Leach, Neil. Rethinking Architecture: A Reader in Cultural Theory. New York: Routledge, 1997. Print.

McLuhan, Marshall. Understanding Media: The Extensions of Man. Routledge [u.a., 2006.

Narula, Uma. Communication Models. New Delhi, India: Atlantic, 2006. Print.

Nesbitt, Kate. Theorizing a New Agenda for Architecture: An Anthology of Architectural Theory 1965-1995. New York: Princeton Architectural Press, 1998.

Nöth, Winfried. Handbook of Semiotics. Bloomington [u.a.: Indiana Univ. Press, 1998.

Nöth, Winfried. Semiotics of the Media: State of the Art, Projects, and Perspectives. Berlin [u.a.: Mouton de Gruyter, 1997.

Rendell, Jane. Art and Architecture: A Place between. London u.a.: I. B. Tauris, 2006.

Schwarzer, Mitchell. Zoomscape: Architecture in Motion and Media. New York: Princeton Archit Pr [u.a., 2004. 


\section{List of Figures:}

\section{Chapter 1: Proof of the Problem}

Fig 1.1] Building Axonometric Analysis, Wellington School of Architecture, Auckland School of Architecture, Yale School of Architecture: Author adapted from, Bing Maps.

Fig 1.2] Building Axonometric Analysis, Minnesota School of Architecture, Harvard School of Architecture, Illinois Institute of Technology: Author adapted from, Bing Maps.

\section{Chapter 2: Proof of the Solution}

Fig 2.1] Carpenter Centre designed by Le Corbusier in 1963: Source, "The Lecorbusier Guide"

Fig 2.2] Kunsthal Model: Source, "Oma: The Construction of Merveilles"

Fig 2.3] The Museu de Arte de São Paulo (1960) designed by Lina Bo Bardi: Source, www.aprenda 450anos.com.br/450anos/img/livro/2548B-(MASP_1969-2000).jpg

Fig 2.4] Museum on the Seashore (1951) designed by Lina Bo Bardi: Source, http://25.media.tumblr. com/tumblr_mab330Wpt11rckws3o1_1280.jpg

Fig 2.5] Cais das Artes (2008) designed by Paulo Mendes da Rocha: Source, http://ad009cdnb. archdaily.net/wpcontent/uploads/2011/12/1324522279-cais-cam3-noturna-final.jpg

Fig 2.6] College of Architecture and Urbanism of the University of São Paulo (1968) designed by Joao Batista Vilanova Artigas, Source: http://www.mimoa.eu/images/10435_l.jpg

\section{Chapter 3: Design}

Fig 3.1 Diagrammatic mapping of identified site: Author adapted from, Google Earth.

Fig 3.2 Building Axonometric: Author's Collection

Fig 3.3] Axonometric of identified site: Author adapted from, Bing Maps.

Fig 3.4] Diagrammatic mapping of identified site: Author's Collection

Fig 3.5] Diagrammatic mapping of identified site: Author's Collection

Fig 3.6] Diagrammatic mapping of identified site: Author's Collection

Fig 3.7] Site plan that depicts the designed site: Author's Collection

Fig 3.8] Site plan that depicts circulation: Author's Collection

Fig 3.9] Site plan that depicts geometry: Author's Collection

Fig 3.10] Site plan that depicts exchange points: Author's Collection

Fig 3.11] Site axonometric that depicts geometry: Author's Collection

Fig 3.12] Building Axonometric: Author's Collection

Fig 3.13] Building Axonometric: Author's Collection 
Fig 3.14] Site axonometric that depicts park and gallery space: Author's Collection

Fig 3.15] Site axonometric that depicts urban amphitheatre: Author's Collection

Fig 3.16] Diagrammatic mapping that depicts formal logic: Author's Collection

Fig 3.17] Diagrammatic mapping that depicts formal logic: Author's Collection

Fig 3.18] Building diagram that depicts formal logic: Author's Collection

Fig 3.19] Diagrammatic mapping that depicts program composition: Author's Collection

Fig 3.20] Exploded Axonometric: Author's Collection

Fig 3.21] Gallery Space: Author's Collection

Fig 3.22] Section: Author's Collection

Fig 3.23] Ground Floor: Author's Collection

Fig 3.24] Sub Floor: Author's Collection

Fig 3.25] Section: Author's Collection

Fig 3.26] Circulation Perspective: Author's Collection

\section{Chapter 4: Critical Appraisal}

Fig 4.1] Communication Diagram: Author adapted from, Shannon-Weaver model of information theory. 
